

\title{
Large distribution and high sequence identity of a Copia-type retrotransposon in angiosperm families
} Elaine Silva Dias, Clémence Hatt, Perla Hamon, Serge Hamon, Michel Rigoreau, Dominique Crouzillat, Claudia Marcia Aparecida Carareto, Alexandre De Kochko, Romain Guyot

\section{To cite this version:}

Elaine Silva Dias, Clémence Hatt, Perla Hamon, Serge Hamon, Michel Rigoreau, et al.. Large distribution and high sequence identity of a Copia-type retrotransposon in angiosperm families. Plant Molecular Biology, 2015, 89 (1-2), pp.83-97. 10.1007/s11103-015-0352-8 . ird-01225496

\section{HAL Id: ird-01225496 \\ https://hal.ird.fr/ird-01225496}

Submitted on 6 Nov 2015

HAL is a multi-disciplinary open access archive for the deposit and dissemination of scientific research documents, whether they are published or not. The documents may come from teaching and research institutions in France or abroad, or from public or private research centers.
L'archive ouverte pluridisciplinaire HAL, est destinée au dépôt et à la diffusion de documents scientifiques de niveau recherche, publiés ou non, émanant des établissements d'enseignement et de recherche français ou étrangers, des laboratoires publics ou privés. 


\section{Large distribution and high sequence identity of a Copia-type retrotransposon in angiosperm families}

Elaine Silva Dias ${ }^{1,3}$ (elainedias_bio@yahoo.com.br)

Clémence Hatt ${ }^{1}$ (clemhatt@gmail.com)

Serge Hamon ${ }^{1}$ (serge.hamon@,ird.fr)

Perla Hamon¹ (perla.hamon@ird.fr)

Michel Rigoreau² (michel.rigoreau@,rdto.nestle.com)

Dominique Crouzillat ${ }^{2}(\underline{\text { dominique.crouzillat@,rdto.nestle.com })}$

Claudia Marcia Aparecida Carareto³ (carareto@ibilce.unesp.br)

Alexandre de Kochko (alexandre.dekochko@ird.fr)

Romain Guyot ${ }^{4 *}$ (romain.guyot@,ird.fr)

${ }^{1}$ IRD UMR DIADE, EVODYN, BP 64501, 34394 Montpellier Cedex 5, France

${ }^{2}$ Nestlé R\&D Tours, 101 AV. G. Eiffel, Notre Dame d'Oe', BP 49716 37097, Tours, Cedex 2,

France

${ }^{3}$ UNESP - Univ. Estadual Paulista, Department of Biology, São José do Rio Preto, SP, Brazil.

4 IRD UMR IPME, COFFEEADAPT, BP 64501, 34394 Montpellier Cedex 5, France

*Corresponding Author: Romain Guyot, Institut de Recherche pour le Développement (IRD), UMR IPME, BP 64501, 34394 Montpellier Cedex 5, France, +33467416455, romain.guyot@ird.fr

\section{Number of Figures: 5}

\section{9,913 words}

Number of Tables: 3

\section{Supplementary file}




\begin{abstract}
Retrotransposons are the main component of plant genomes. Recent studies have revealed the complexity of their evolutionary dynamics. Here, we have identified Copia25 in Coffea canephora, a new plant retrotransposon belonging to the Ty1-Copia superfamily. In the Coffea genomes analyzed, Copia25 is present in relatively low copy numbers and transcribed. Similarity sequence searches and PCR analyses show that this retrotransposon with LTRs (Long Terminal Repeats) is widely distributed among the Rubiaceae family and that it is also present in other distantly related species belonging to Asterids, Rosids and monocots. A particular situation is the high sequence identity found between the Copia25 sequences of Musa, a monocot, and Ixora, a dicot species (Rubiaceae). Our results reveal the complexity of the evolutionary dynamics of the ancient element Copia25 in angiosperm, involving several processes including sequence conservation, rapid turnover, stochastic losses and horizontal transfer.
\end{abstract}


4 Authors and Affiliations

5

6 Elaine Silva Dias ${ }^{1,3}$ (elainedias_bio@yahoo.com.br)

7 Clémence Hatt ${ }^{1}$ (clemhatt@gmail.com)

$8 \quad$ Serge Hamon ${ }^{1}$ (serge.hamon@ird.fr)

$9 \quad$ Perla Hamon ${ }^{1}$ (perla.hamon@ird.fr)

10 Michel Rigoreau² (michel.rigoreau@rdto.nestle.com)

11 Dominique Crouzillat ${ }^{2}$ (dominique.crouzillat@,rdto.nestle.com)

12 Claudia Marcia Aparecida Carareto³ (carareto@ibilce.unesp.br)

13 Alexandre de $\mathrm{Kochko}^{1}$ (alexandre.dekochko@,ird.fr)

14 Romain Guyot ${ }^{4 *}$ (romain.guyot@,ird.fr)

15

${ }^{1}$ IRD UMR DIADE, EVODYN, BP 64501, 34394 Montpellier Cedex 5, France

17 2Nestlé R\&D Tours, 101 AV. G. Eiffel, Notre Dame d'Oe', BP 49716 37097, Tours, Cedex 2,

18 France

$19{ }^{3}$ UNESP - Univ. Estadual Paulista, Department of Biology, São José do Rio Preto, SP, Brazil.

$20{ }^{4}$ IRD UMR IPME, COFFEEADAPT, BP 64501, 34394 Montpellier Cedex 5, France

21

22 *Corresponding Author: Romain Guyot, Institut de Recherche pour le Développement (IRD),

23 UMR IPME, BP 64501, 34394 Montpellier Cedex 5, France, +33467416455,

24 romain.guyot@ird.fr

25 Data deposition: KM439056 to KM439101 


\section{Abstract}

2

3 Retrotransposons are the main component of plant genomes. Recent studies have revealed the

4 complexity of their evolutionary dynamics. Here, we have identified Copia25 in Coffea

5 canephora, a new plant retrotransposon belonging to the Tyl-Copia superfamily. In the

6 Coffea genomes analyzed, Copia 25 is present in relatively low copy numbers and transcribed.

7 Similarity sequence searches and PCR analyses show that this retrotransposon with LTRs

8 (Long Terminal Repeats) is widely distributed among the Rubiaceae family and that it is also

9 present in other distantly related species belonging to Asterids, Rosids and monocots. A

10 particular situation is the high sequence identity found between the Copia25 sequences of

11 Musa, a monocot, and Ixora, a dicot species (Rubiaceae). Our results reveal the complexity of

12 the evolutionary dynamics of the ancient element Copia25 in angiosperm, involving several

13 processes including sequence conservation, rapid turnover, stochastic losses and horizontal

14 transfer.

15

16

17 Keywords

18

19 Copia25, transposable element, genome dynamics, sequence conservation, horizontal transfer,

20 Rubiaceae.

21 


\section{Introduction}

2

3 Transposable elements (TEs) are the major component of plant genomes. TEs are typically

4 "vertically" transmitted from parent to offspring. If a new insertion occurs in germ cells

5 tissues, the new copy will be transmitted to the progeny. In certain cases, TEs can be

6 horizontally transferred (HT) between reproductively isolated species. Although more than

7200 cases of HT have been reported most of them involve animals (Schaack et al. 2010),

8 mainly insects (mostly Drosophila), and few potential cases have been reported in plants

9 (Cheng et al. 2009; Diao et al. 2006; Fortune et al. 2008; Roulin et al. 2008) with the 10 exception of a very recent observation (E1 Baidouri et al. 2014). The HTs concern both Class I

11 (or Retrotransposon) and Class II (or Transposons) elements, and the mechanisms underlying

12 TE HTs remain speculative in most of the cases (vectors could be pathogens, intracellular

13 parasites, insects, etc.). Because TEs play a major role in the dynamics of genomes, their

14 direct introduction into a "naïve" genome through HT may induce important consequences in

15 chromosomal and genomic evolution. However, the detection of potential HT of TEs in

16 complete genomes is relatively complex and requires highly sensitive methods to differentiate

17 between unresolved sequence conservation and HT events (de Carvalho and Loreto 2012). In

18 the absence of a clear mechanism underlying HT, cases of outstanding sequence conservation

19 of TEs between evolutionarily distant plant species living in separate geographical areas have

20 raised questions as to the existence of other mechanisms leading to this conservation (Moisy

21 et al. 2014). The recent availability of plant genome sequences (Michael and Jackson 2013)

22 gave new opportunities to identify and to characterize transposable elements and to gain a

23 higher understanding of the evolutionary dynamics of these elements and their conservation

24 between distantly related species. 
The coffee genus (Coffea) that belongs to the Rubiaceae family, comprises 124

2 species, originating from Africa, Madagascar, the Mascarene Islands, Asia and Oceania

3 (Davis 2010; Davis 2011). Coffea species are diploids $(2 \mathrm{n}=2 \mathrm{x}=22)$ and generally

4 allogamous. The notable exception is the self-fertilizing allotetraploid Coffea arabica $(2 \mathrm{n}=$

$54 \mathrm{x}=44$ ), native to the Ethiopian highlands and originating from a recent hybridization of two

6 different diploid ancestors, C. canephora and C.eugenioides (Lashermes et al. 1999; Yu et al.

7 2011). The current possibility of accessing genomic and transcriptomic sequences of Coffea

8 species has made it possible to expand our knowledge of the composition and behavior of TEs

9 in these important species. The analysis of the $C$. canephora genome showed that these 10 sequences contained about $50 \%$ of transposable elements (Denoeud et al. 2014). The vast majority of them (85\%) are retrotransposons with LTRs (LTR-RTs). The study of TEs in

12 Coffea is very recent and the few individual TEs investigated to date show different dynamics between closely related coffee species (Hamon et al. 2011; Yuyama et al. 2012).

In this study, LTR-RTs were identified in the C. canephora genome using BAC-end sequences (BESs) and 454 sequences. One of them, a Ty1-Copia element named Copia25, was characterized and analyzed under different aspects of its evolution because its nucleotide

17 sequence showed unusually high similarities with distantly related plant genomes. Furthermore, Copia25 was found quite similar to Rider, an active retrotransposon identified in the tomato with a rather unique evolutionary history. Rider activity has played a role in the origin of at least three different phenotypes of this species (Jiang et al. 2009; Jiang et al. 2012;

21 Xiao et al. 2008). Since it is absent in Solanum tuberosum, it has been suggested that Rider 22 appeared in the tomato by HT from Arabidopsis thaliana (Cheng et al. 2009). The similarity shared between Copia25 and Rider makes the TE identified in C. canephora interesting to 24 investigate, particularly for its activity and evolutionary dynamics. In the current study, we 25 show that Copia25 is an active element in Coffea, widely present in Rubiaceae species. In 
1 addition, a phylogenetic analysis indicates outstanding conservation of Copia25 in coffee

2 trees and in distantly related species, such as banana (Musa genus), a monocot. The different

3 processes that can lead to high conservation of Copia25 in Angiosperms are discussed.

4

5

6 Materials and Methods

7

8 Genome sequencing

9

10 The Next-Generation Sequencing (NGS - by Genomic 454 Pyrosequencing - GS Junior

11 System Roche) was performed in two accessions of $C$. canephora Pierre ex A. Froehner

12 (HD200-94 a double haploid from the Congolese diversity group, also used for whole genome

13 sequencing - Denoeud et al. 2014, http://coffee-genome.org -, and BUD15 from Uganda), as

14 well as in one accession from each of the following taxa: C. arabica L. (ET39 from Ethiopia),

15 C. eugenioides S. Moore (DA56 from Kenya), C. pseudozanguebariae Bridson (08107 from

16 Kenya), C. heterocalyx Stoff (JC65 from Cameroon), C. racemosa Lour (IA56 from

17 Mozambique), C. humblotiana Baill (A.230 from Comoros), C. millotii J.-F. Leroy (ex-

18 dolichophylla, A.206 from Madagascar) and C. tetragona Jum. \& H. Perrier (A.252 from

19 Madagascar), Coffea (ex-Psilanthus) horsfieldiana (Miq.) J.-F. Leroy (HOR from Indonesia)

20 and Craterispermum Sp. Novo Kribi (from Cameroon) (Chevalier 1946; Maurin et al. 2007).

21 The cultivars and the above-mentioned sequenced accessions grow in the IRD greenhouses

22 (Montpellier, France), at the Kianjavato research station (Madagascar) or in the Nestlé R\&D

23 greenhouses (Tours, France). The total genomic DNA was extracted from young leaves using

24 the Qiagen DNeasy Plant Mini Kit following the manufacturer's protocol. The library and

25 sequencing for the NGS were performed at the Nestlé R\&D laboratory according to the 
1 Roche/454 Life Sciences Sequencing Method. Data were submitted to GenBank, BioProject

2 PRJNA242989.

3

4 Sequence Analyses

5

6 We used 131,412 BAC end sequences (BESs) (Dereeper et al. 2013) obtained by Sanger 7 sequencing and 106,459 sequences obtained by 454 Roche-NGS technology, both derived

8 from the C. canephora HD200-94 accession. All sequences (Sanger and 454 Roche) were 9 used for the assembly using AAARF (Assisted Automated Assembler of Repeat Families 10 DeBarry et al. 2008). The following parameters for the BLAST analyses and the Minimally

11 Covered Sequences (MCS) construction and controlling "build" extensions were applied:

12 minimum hit length: 150; minimum hit identity: 0.89; minimum coverage depth: 4; required

13 MCS length: 150; maximum E-value: $1 \mathrm{e}^{-25}$; required coverage length: 150; minimum hit

14 number: 2; required overlap between MCS and new query: 90; and maximum times a number

15 sequence is used in each direction: 13. These parameters were those that gave best assembly results after several modification and assembly testing.

AAARF "builds" were analyzed using BLASTx (min E-value $1 \mathrm{e}^{-4}$ ) against public protein sequence databases (uniprot_sprot; http://www.uniprot.org/), and transposable element databases available in Repbase (Jurka et al. 2005 - http://www.girinst.org/repbase/) and Gypsy DB 2.0 (http://gydb.org - Llorens et al. 2011). The graphical dot-pot (Dotter Sonnhammer and Durbin 1995) was also performed. The final annotations of each "build" were edited in Artemis (Carver et al. 2005). Validation of LTR-RT "build" structures was performed by comparative analysis with public Coffee BAC sequences, from the NCBI and the genome of $C$. canephora (Denoeud et al. 2014 - coffee-genome.org). Five BAC clones for C. canephora (EU164537, HQ696512, HQ696507, HQ696513 and HM635075) and 12 BAC 
1 clones for C. arabica (GU123896, GU123899, GU123898, GU123894, GU123897,

2 GU123895, HQ696508, HQ696510, HQ696509, HQ696511, HQ834787 and HQ832564)

3 were downloaded from GenBank, accounting for a total of 3,023 Mb. BLASTN searches (E-

4 value $<1 \mathrm{e}^{-150}$ ) against public Expressed Sequenced Tags (ESTs) databases from C. canephora

5 and $C$. arabica were used to evaluate the transcription of the builds.

6

$7 \quad$ Estimation of the Copia25 copy number using 454 sequencing survey

8

9 BLASTN searches were carried out with the full-length Copia25 sequence (from BAC 10 HQ696507) as query. Reads with more than $90 \%$ of nucleotide identity with Copia25 over a 11 minimum of $80 \%$ of the read lengths were considered as potential fragments of the element.

12 Cumulative lengths of aligned reads to Copia25 were used to extrapolate the contribution of 13 the element to each genome size investigated.

\section{Identification of Copia25 in plant genomes}

17 The sequence trimmed from AAARF was blasted against the C. canephora genome, as well as against 40 angiosperm and one non-angiosperm genome sequences available in the public databases of NCBI, Phytozome and Gramene (Table S1). BLASTN was used to search for the complete nucleotide sequence or the coding region of Copia 25 in the genomes. The retrieved sequences were analyzed using LTRharvest (Ellinghaus et al. 2008) in order to recover only

22 the sequences with a structure similar to retrotransposons. These sequences were compared to 23 the amino acid sequence of the Copia25 reverse transcriptase (RT) using TBLASTN and 24 against the Tyl-Copia retrotransposon databases of plants (Repbase http://www.girinst.org) 25 resulting in 98 sequences from 34 species (Table S2). 


\section{Molecular analysis}

4 The DNA of 24 Rubiaceae species (Table S3, Fig. S1) was extracted by using DNeasy Plant

5 mini-kit (QIAGEN). The DNA of the Musa species was donated by Dr. A. D'hont (CIRAD,

6 France). Primers were designed on intact RT region of C. canephora Copia25 genomic

7 sequences using Primer3 (http://bioinfo.ut.ee/primer3-0.4.0/primer3/) (Forward: 5' GGG

8 GTT GAA GAT GCA AGG TA 3'; Reverse: 5' AGC TGC TCC CAA ATC TTT CA 3'). For

9 the reaction, 0.625 unit of Taq polymerase (Invitrogen), $20 \mathrm{ng}$ genomic DNA, $1 \mathrm{mM}$ of

$10 \mathrm{MgCl}_{2}, 1 \mathrm{X}$ buffer, $0.08 \mathrm{mM}$ of dNTPs and $0.4 \mathrm{mM}$ of each primer were used for a final

11 volume of $25 \mu \mathrm{L}$. PCR conditions were as follows: initial denaturation $\left(94{ }^{\circ} \mathrm{C}, 120 \mathrm{~s}\right)$;

12 followed by 40 cycles of denaturation $\left(94^{\circ} \mathrm{C}, 30 \mathrm{~s}\right)$, annealing $\left(55^{\circ} \mathrm{C}, 30 \mathrm{~s}\right)$ and extension $(72$

$\left.13{ }^{\circ} \mathrm{C}, 180 \mathrm{~s}\right)$. Each PCR product was analyzed by gel electrophoresis on $1.2 \%$ agarose gel, 14 purified (DNA GFX DNA \& Gel Band, GE) and cloned (TOPO XL Cloning kit, Invitrogen) 15 according to the manufacturer specifications. The plasmids extracted were sequenced using 16 the specific primers. The Copia25 sequences were registered under the GenBank Accession

17 Numbers KM439056 to KM439101. For the reverse transcription polymerase chain reaction (RT-PCR) $1 \mu \mathrm{g}$ of the total RNA from leaves of C. canephora, C. eugenioides and C. arabica was treated with RQ1 RNase-Free DNase (Promega) and reverse-transcribed using ImPromII ${ }^{\mathrm{TM}}$ Reverse Transcription System (Promega). The synthesized cDNA served as templates for RT-PCR. DNA contamination was checked using the primers of the gene sucrose synthase

22 (SUS10/SUS11 - Marraccini et al. 2011). RT-PCR was performed using the same specific 23 primers according to the protocol described as before, with $50 \mathrm{ng}$ of cDNA.

\section{Evolutionary Analyses}


2 Phylogenetic analyses were performed with MEGA 5.2 (Kumar et al. 2008) on sequence

3 datasets aligned with the MAFFT program. Each phylogeny was reconstructed using the best

4 model using Find Best DNA/Protein Model (Maximum Likelihood) in Mega 6 (Tamura et al.

5 2013), with 1000 replicates; the bootstrap consensus tree inferred is taken to represent the

6 evolutionary history of the taxa analyzed. All positions containing gaps and missing data were

7 eliminated. As rates of synonymous substitution are not available for Rubiaceae (genes or

8 TEs), and because LTR sequences (non-coding regions) and those from the RT domain

9 (coding region) may evolve differently, two rates, estimated for grasses and palms, were used.

10 The age of insertion of Copia25 within C. canephora genome was estimated using the 11 molecular clock equation, as previously described (Moisy et al. 2014; SanMiguel et al. 1998;

12 Wicker and Keller 2007), where $k$ was the Kimura 2-parameter distance between both LTRs

13 of the same copy, and $r$ is $1.3 \times 10^{-8}$ base substitutions per site per year (Ma and Bennetzen

14 2004). The Kimura 2-parameter method of distance estimation of non-coding nucleotide

15 sequences was used for LTR distance estimation (SanMiguel et al. 1996). However, gene

16 conversion between LTR of the same element could be a source of errors in estimating

17 insertion time. This putative error is not taken into account in our analysis since conversion of

18 LTR remains poorly understood in plant genomes. The age of the ancestor of the Copia25

19 sequences was also estimated using the molecular clock equation, using Ks (number of

20 synonymous substitutions per synonymous site) and the rate of synonymous substitutions as

$216.5 \times 10^{-9}$ base substitutions per site per year (Gaut et al. 1996) for the RT domain (Vitte et al.

22 2007).

23 In order to investigate whether Copia25 was under selective pressure a codon substitution

24 model was used to estimate $\omega(\mathrm{Ka} / \mathrm{Ks})$. The $\omega$ ratio measures the direction and the magnitude

25 of selection on amino acid changes, with values of $\omega<1,=1$, and $>1$ indicating negative 
1 purifying selection, neutral evolution, and positive selection, respectively. To estimate $\omega$ two approaches were used: (i) the $\mathrm{Ka} / \mathrm{Ks}$ pairwise ratio for species with the full-length polyprotein sequence available (coffee, potato, tobacco and banana); and (ii) likelihood ratio tests (LRTs)

4 for a simplified phylogeny (Fig. S2) containing species representatives of each of the

5 Rubiaceae tribes and potato, tobacco and banana, using $315 \mathrm{nt}$ of the RT domain. Premature

6 stop codons were removed from the sequences for both analyses. For the pairwise $\mathrm{Ka} / \mathrm{Ks}$, the

7 reference sequences of the Copia25 Subfamilies 1 and 2 (chr7_16264485-16269785 and chr8_8081742-8086630 respectively) were compared with their homologous sequences in

9 potato, tobacco and banana. $K a$ and $K s$ were obtained using DnaSP v5 (Librado and Rozas

10 2009). Selective pressure acting on COSII (conserved orthologs group) genes of potato,

11 banana and coffee (Wu et al. 2006) was also investigated. The COSII sequences in potato and

12 C. canephora are available on the Sol Genomics Network website (http://solgenomics.net).

13515 COSII accessions present in single copy in potato and coffee were blasted (BLASTn)

14 against the Musa acuminata CDSs (D'Hont et al. 2012 - http://banana-genome.cirad.fr/) in

15 order to obtain the Musa COSII sequences. Seven COSII sequences showing the highest

16 sequence identity were used to calculate the $\mathrm{Ka} / \mathrm{Ks}$ ratio and nucleotide identity (Table $\mathrm{S} 4$ ).

17 The second approach used different $\omega$ ratio parameters for different branches on the 18 phylogeny (Anisimova and Ziheng 2007; Yang and Nielsen 1998). To estimate the log 19 likelihood values (LRT), a one-ratio model was used. This model assumes the same $\omega$ free or 20 fixed $(\omega=1)$ parameter for the entire tree, Model I and Model II, respectively. A two-ratio 21 model was used to estimate the LRTs for specific clades on the phylogeny, since we assumed 22 that the sequence group of interest (separately for Ixora, Model III $=\omega$ free, and Model IV $=$ $23 \omega$ fixed; and, for Musa, Model V $=\omega$ free, and Model VI $=\omega$ fixed) has a different $\omega_{\mathrm{F}}$ from 24 that of the $\omega_{\mathrm{B}}$ background. For the pairs of models (I $v s$ II, III $v s$ IV, V vs VI)), the $\log$ 
1 likelihood values were compared in a hypothesis test $\left(X^{2}\right)$. These analyses were implemented using the codeml program in the PAML package (Yang 1997).

3

4

$5 \quad$ Results

6

Assembly of repeated sequences with BAC-end Sanger sequences and 454 random reads

from C. canephora

9

Sanger and 454 sequences from C. canephora (accession HD200-94) were used to identify and characterize the TEs. Two bacterial artificial chromosome (BAC) libraries were recently constructed from the same plant and a total of 134,827 Sanger sequences (mean size $683 \mathrm{bp}$ ) were generated from BAC-end sequences (BES) and released (Dereeper et al. 2013). In addition, 106,459 random 454 Roche reads (mean size 423 bp) were also generated from the same plant (Table S5).

In all, Sanger and 454 sequences represent 137,104,866 bp (241,286 sequences),

17 giving an estimated coverage of $19.5 \%$ of the $C$. canephora genome $(710 \mathrm{Mb})$. They were used together to assemble repeated sequences using the Assisted Automated Assembler of Repeat Families Algorithm (AAARF, DeBarry et al. 2008). A total of 1,306 "builds" (also called contigs) were generated with a length ranging from 135 to $24,745 \mathrm{bp}$, and a mean length of $1,306 \mathrm{bp}$. Most of them (45\%) have a length comprised between 0.5 and $1 \mathrm{~kb}$. In total, 317 builds showed similarities with TE proteins available in public databases after translating the assembled sequences. Fifty-two of them, showing sizes larger than $3 \mathrm{~kb}$, were selected for the subsequent analysis. Forty-nine out of 52 showed strong similarity to LTR-RT proteins (Table S6 and Table S7). Over the 49 contigs, 12 elements were removed due to non- 
canonical (complex) structure, suggesting incorrect assembly, and in a significant number of builds, manual corrections were made (Table S6, 10 builds labeled with $\ddagger$ ), following the same procedure as described in De Barry et al. (2008). The 37 remaining builds with canonical TE structures showed exclusively similarities with LTR-RT proteins, suggesting that it may represent the main abundant transposable element family in the $C$. canephora genome (Table 1). These 37 potential retrotransposon builds, were manually annotated, and incomplete structures of all them were found (Fig. S3). According to the structural annotation, the were classified as "LTR-I-LTR" when the internal region and both complete or partial LTRs were present; as, "I" if only an internal region was present, as "LTR-I" with complete or partial 5' LTR with an internal region, and, "I-LTR" with an internal region and complete or partial 3' LTR (Table S6).

The 37 LTR-RT builds were used as query for similarity search (BLASTn) for complete or partial copies present in the available Coffea BAC clones sequences (Table S6). Ten LTR-RT builds showed high levels of nucleotide conservation with nine $C$. canephora (4) and C. arabica (5) BAC sequences (BLAST E-value cutoff: 10e ${ }^{-100}$; Table S6). Moreover, some builds showed similarities with Coffea transcriptomic sequences. Indeed, 15 and four LTR-RT builds were found in C. canephora and C. arabica ESTs, respectively (Table S7).

\section{Characterization of Copia25, a Ty1-Copia LTR retrotransposon in Coffee trees}

Among the retrotransposons identified in C. canephora sequences (accession HD200-94), the sequence of one Ty1-Copia element, hereafter named Copia25, showed high BLASTN scores across various distantly related plant genomes, suggesting that Copia25 has a singular evolutionary history. Copia25 also showed an overall structure similarity to Rider (EU195798), an active retrotransposon found conserved between distant dicot species (Cheng 
et al. 2009; Jiang et al. 2012), as indicated by dot-plot alignment (not shown). The Copia25

2 reassembled contig was blasted (BLASTN $10 \mathrm{e}^{-100}$, Table S6) against $C$. arabica and $C$.

3 canephora BAC sequences. It was found in $C$. canephora but with an uncommon arrangement, which appears to be a tandem of two elements sharing one LTR sequence in the median of the structure (accession HQ696507). In C. arabica, in turn, a complete sequence of

65,382 bp was found. This sequence is flanked by two perfect 5-bp TSDs (5'-GGAAC-3'), and

7 its two LTRs are both 530 bp long and show high sequence identity (99.2\%) (accession

8 HQ832564 - Fig. S4). This copy is localized on a homologous region to C. canephora, most

9 probably the $C$. canephora sub-genome within $C$. arabica, but it is absent in the syntenic region in both 126 (Moschetto et al. 1996; Yu et al. 2011) and HD200-94 C. canephora genotypes (Denoeud et al. 2014).

A search was also made for the Copia25 contig (using Censor) in the C. canephora genome (Denoeud et al. 2014) and 72 full-length copies were identified. All of them showed premature stop codons in the pol coding region, indicating that none of them is potentially functional. Nonetheless, similarity searches showed high sequence identity between Copia25 and Expressed Sequence Tags (98 and 99\% of nucleotide identity with DV679393 and GT681881, respectively). In addition, the Copia25 RT regions were successfully amplified by RT-PCR on RNA extracted from C. canephora, C. arabica and C. eugenioides leaves (Fig. S5).

Full-length Copia25 copies exist throughout the $C$. canephora genome mainly in genepoor and LTR-RTs rich areas. The majority of them are located in the non-anchored set of scaffolds (pseudo-chromosome "0") (Fig. 1a; Table S8). The sharing of structural characteristics among group of sequences of a TE family might indicate the occurrence of subfamilies. In such cases, the different groups have different most recent ancestral copy - i.e. different mother (or master) copy -, which independently originated copies. A Maximum 
1 Likelihood with the distance corrected by General Time Reversible model and 1000 replicates

2 phylogenetic tree was produced using the pol (2,640 nt) nucleotide sequence of the 72 full-

3 length Copia25 copies. Based on the tree topology, two clusters were segregated (Fig. 1b).

4 Following Wicker's parameters (Wicker et al. 2007) segregating criterion they are hereafter

5 considered as subfamilies, one harboring 44 copies (Subfamily 1) and the other 28 (Subfamily

6 2). Only one copy did not group with either of the two clusters; this copy was discarded from

7 further analyses. In each subfamily, the sequence with the perfect structure (based on the best 8 conservation of both LTRs and the presence of an intact or few stop codons in the ORF 9 coding for the polyprotein) was chosen as a reference sequence for the subfamily (Subfamily 10 1: chr7_16264485-16269785; Subfamily 2: chr8_8081742-8086630). These two sequences 11 are $87.8 \%$ identical, and have $9.8 \%$ of InDels. The differences between them are mainly 12 concentrated in the LTR region, where the identity is only $71 \%$, and InDels reach $15 \%$, 13 resulting in only $59 \%$ of overlap. Such difference results in poor LTR alignment of the 72 copies. Additionally, Subfamily 2 presents a 208 bp deletion in the UTL 5' (Untranslated Leader) region. The corrected distances (Tamura-3 parameters) within each subfamily are 0.123 and 0.138 respectively, for Subfamily 1 and 2, and 0.222 between subfamilies (overall mean of 0.174$)$. The divergence between the two LTRs of each copy was calculated and an insertion time was inferred. Subfamily 1 showed a mean time of insertion of $2.97 \pm 0.204$ Mya (minimum: 0.5, maximum: 5.2 Mya) and Subfamily 2 showed a mean time of insertion of $4.53 \pm 0.399$ Mya (minimum: 1.3, maximum 10.1 Mya) (Fig. 2, Table S8).

21

\section{Presence of Copia25 in the Rubiaceae family}


1 species. First, 11 genotypes representing 10 Coffea species (including ex-Psilanthus) and

2 Craterispermum sp. Novo kribi were surveyed using high-throughput 454 Roche sequencing.

3 The number of bases produced for each species and the estimated genome coverage according

4 the genome sizes are shown in the Table 2. The 454 sequences were used to survey the

5 presence of highly conserved Copia 25 sequences, using as criteria: $90 \%$ minimal nucleotide

6 identity over $80 \%$ of the sequence length. The number of Copia 25 conserved sequences found

7 for each species and their respective cumulative length according to the genome size are

8 available in the Table 2. Sequences fitting these criteria were present in all Coffea genomes

9 studied here, but not in Craterispermum. The cumulative length of Copia25 reads was estimated to range from 186 to $1,513 \mathrm{~kb}$ of estimated cumulative sequences in diploid species and $842 \mathrm{~kb}$ in the allotetraploid C. arabica (Table 2).

The presence of Copia25 was also investigated by PCR amplification and sequencing of the product in 13 Coffea and 11 other Rubiaceae species (Table S3, Fig. S1). The Copia25 RT region was amplified and sequenced in 13 Coffea species, three from West Africa $(C$. stenophylla, C. humilis and C. ebracteolatus), one from West/Central Africa (C. canephora), three from East Africa (C. costatifructa, C. pseudozanguebariae and C. eugenioides), one

17 from Northeast Africa (C. arabica), and five from Indian Ocean Islands (C. millotii - exdolichophylla -, C. perrieri, C. resinosa, C. tetragona and C. vianneyi) (Chevalier 1946;

19 Maurin et al. 2007). The same region was also amplified and sequenced in 11 other Rubiaceae species: Bertiera iturensis, Tricalysia congesta, Oxyanthus formosus, Ixora sp., I. coccínea, I. finlaysoniana, I. foliicalyx, Polysphaeria parvifolia, Coptosperma sp., Pyrostria sp., and Craterispermum schwenfurthii. The final dataset contains 319 nucleotides, and the nucleotide identity varied from $62 \%$ to $100 \%$ among different sequences comparisons (Table S9). 
Besides the Rubiaceae species, similar Copia25 sequences were sought among the 40 available plant sequences representing the angiosperm clades, and one non-angiosperm species using BLASTN. Similar Copia25 sequences were found in 34 species but not in the remaining eight ones, as follows: Arabidopsis lyrata, Carica papaya, Cucumis sativus, Fragaria vesca, Linum usitatissimum, Selaginella moellendorffii, Phoenix dactylifera and Zea mays (Table S1).

In the 34 genomes where sequences similar to Copia25 were found, these latter were extracted for further phylogenetic analysis. Using a fragment of $750 \mathrm{bp}$ from the RT region, a phylogeny was reconstructed using Maximum Likelihood, with the distance corrected by Tamura 3-parameter and 1000 replicates in order to investigate the relationships among the Copia25 sequences (Fig. 3, Fig. S6 and Tables S10 and S11). One well-supported (95\% bootstrap value) phylogenetic clade was found to include C. canephora Copia25 and sequences belonging to four dicotyledonous species: Nicotiana benthamiana, N. tabacum, $S$. tuberosum (Solanaceae) and Ricinus communis (Euphorbiaceae), and more surprisingly, three monocotyledonous species, Musa accuminata and M. balbisiana (Musaceae), and, in a basal position, Eleais guineensis (Arecaceae). These sequences were considered homologous to Copia 25 because they share over $80 \%$ sequence identity over $80 \%$ of their length in the reverse transcriptase domain (Wicker et al. 2007), except for $R$. communis and E. guinensis.

21 Since these two species cluster within the clade and share, with Copia 25 , over $70 \%$ of identity they were considered to belong to the same family. strongly-supported clades composed of species of the same family, which supports a hypothesis of vertical inheritance (Fig. 3). It is the case of the elements found in the 
1 monocotyledonous family of Poaceae where all of them cluster in a clade with a 94\%

2 bootstrap value. A similar occurrence was found in the Malvaceae species $(100 \%)$ and in

3 Fabaceae (98\%) species, but it is also weakly supported among Brassicaceae (79\%). The

4 exceptions in this context are the particular strongly-supported relationships between

5 Medicago truncatula (Fabaceae) and Mimulus guttatus (Phrymaceae) (94\%), among Populus

6 trichocarpa (Salicaceae), Gossypium hirsutum (Malvaceae) and Malus domestica (Rosaceae)

7 (100\%), and finally between Solanum lycopersicum (Solanaceae) and Arabidopsis thaliana

8 (Brassicaceae) $(100 \%)$

The reconstructed phylogeny using only sequences recovered from public databases

10 (Fig. 3) did not show a clear relationship between the sequences from coffee tree and those

11 from other species in the clade. In an effort to better understand the relationships of Copia25

12 among the species present in the Copia25 clade, we reconstructed a new Maximum

13 Likelihood phylogeny (with the distance corrected by Tamura 3-parameter and 1000

14 replicates), adding RT sequences obtained from several Rubiaceae species and three

15 Musaceae species (M. accuminata, M. balbisiana and M. boman) (Fig. 4 and Fig. S7). As

16 shown in Fig. 4, the unrooted phylogenetic tree revealed that Copia25-Musa is nested into the

17 Rubiaceae species as shown by a closer well-supported relationship (bootstrap value 92\%) between Copia25-Musa and Copia25-Ixora and between Craterispermum sp. and all

19 Rubiaceae and Musaceae species (bootstrap value 65\%). Rubiaceae and Musaceae Copia25

20 are clearly separated from Solanaceae by high bootstrap value (92) and a topology structure.

21 This result suggested that Rubiaceae and Musaceae Copia25 constitute a unique evolutionary 22 lineage (Fig. 4).

23 To further confirm the close relationship between Copia25-Coffea and Copia25-Musa,

24 we first aligned each Copia25-Coffea sequence (Copia25 C. canephora reference sequence of 25 Subfamily 1 and 2) with the Copia25-Musa (M. balbisiana AC186755). The alignments 
1 showed an overall nucleotide identity of $74.1 \%$ and $79.6 \%$ for Subfamily 1 and 2,

2 respectively, and an overall amino acid sequence identity rate of $81.7 \%$ (similarity: $79.8 \%$ )

3 with Subfamily 1, and 81.60\% (similarity: 80.1\%) with Subfamily 2 (Fig. 5a). Their LTRs

4 were also extracted and aligned, showing a high identity rate (53.9\% between Musa and the

5 reference sequence of Subfamily 1; and 59.4\% with the Subfamily 2 reference sequence) (Fig.

65 b). This level of identity is indeed quite significant for non-coding regions and considering

7 the species divergence, i.e. about 150 Mya (Chaw et al. 2004; Wikstrom et al. 2001).

8 Homologous sequences to Copia25-Musa from the M. balbisiana genome (B genome) were

9 also found in the sequenced M. acuminata genome (A genome; D'Hont et al. 2012). These

10

11

12

13

14

15

16

17

18

19

20

21

22

23

24

25

\section{Evolution of Copia25 in monocots and dicots}

To investigate the evolution of Copia25 in detail, we used the nucleotide sequences of Copia25 from M. balbisiana, C. canephora, S. tuberosum and N. benthamiana for pairwise sequence comparisons. The results summarized in Supplementary Table S12 show higher identity between the Copia25 of coffee and banana than between all the other species. We compared the identity of Copia25 with the identities of seven COSII sequences showing the highest sequence identity between banana and coffee. These genes share an average of $74.7 \%$ of identity between banana and coffee, while the coding region of Copia 25 shows $85 \%$. For the Copia25 polyprotein and these seven COSII genes, we performed a pairwise Ka/Ks (nonsynonymous per synonymous substitution ratio) analysis by comparison of banana, potato, tobacco and coffee sequences. Both COSII and Copia25 were under purifying selection, 
1 however they were found more relaxed in Copia25 (minimum: 0.233, maximum: 0.287) than

2 in COSII (minimum: 0.038, maximum: 0.215) sequences.

3 The LRT results reinforce the proposition of the purifying selection acting on the

4 Copia25 sequences (Table 3). The log likelihood values using a one-ratio model (Model I: $\omega$

5 free, and Model II: $\omega$ fixed) for the entire phylogenetic tree (Fig S2) were significantly lower

6 than the neutral expectation, indicating purifying selection $(0.191,2 \Delta \ell=239.308, \mathrm{p}<0.01)$.

7 The LRTs of the Ixora and Musa clades were estimated separately. For these, a two-ratio

8 model was applied, since we assumed that the sequence group of interest has a different $\omega_{\mathrm{F}}$

9 from that of the $\omega_{\mathrm{B}}$ background (Model III: $\omega$ free, and Model IV: $\omega$ fixed, for Ixora clade;

10 and Model V: $\omega$ free, and Model VI: $\omega$ fixed, for the Musa clade). Purifying selection was

11 also detected for Ixora clade $(0.127,2 \Delta \ell=33.568, \mathrm{p}<0.01)$, while for the Musa clade the $\omega$

12 value did not differ from neutral evolution (Table 3). The negative selective pressure would

13 explain the narrow relationship between the coffee and banana sequences. However, the

14 negative selection for Copia25 and COS, and the neutrality for Copia25 in Musa clade

15 indicate that this alone does not explain their clustering in the phylogeny.

16 The divergence time of two sequences harbored by two species from their common

17 ancestral sequence was estimated by using both COSII and Copia25. The estimated 18 divergence time using Copia25 sequences for Musa and Coffea is much lower than for COSII 19 sequences. While the latter ones ranged from 94.5 to 181.8 Mya, when using Copia25 the 20 time was 35.5 and 31.7 Mya. Indeed, the estimated divergence time using the Copia25 from 21 banana and the Solanaceae species is similar to that found for coffee, tobacco and potato. The 22 high similarity and the $K s$ values for the comparisons between coffee and banana with the other Solanaceae species indicate that the Copia25 sequence could be a recent guest in banana species genome. 
Discussion

3

4 Copia25 in the Rubiaceae family

5

In this study, we identified an expressed Tyl-Copia in the C. canephora genome,

7 Copia25, and analyzed it under various aspects, providing a broad insight into its evolution.

8 Copia25 was found distributed in several species of the Coffea genus from Africa, the Indian

9 Ocean Islands and Indonesia. The occurrence of Copia25 in these species denotes that it could

10 be present in the ancestor of this phylogenetic group and has been inherited by the derived

11 lineages. Our proposition of its presence in the Coffea lineage ancestor is reinforced by the

12 occurrence of Copia25 in at least two of the three subfamilies of the Rubiaceae family,

13 Rubioideae (Craterispermum schwenfurthii) and Ixoroideae (Coffea spp., Ixora spp., Bertiera

14 iturensis, Coptosperma sp., Oxyanthus formosus, Polysphaeria parvifolia, Pyrostria sp.,

15 Tricalysia cloneongesta), also suggesting its ancient evolutionary history in Rubiaceae.

16 Altogether these data suggest the presence of Copia25 in both of the Rubiaceae subfamilies

17 preceding their ancient divergence.

18

19 High sequence identity of Copia25 of over $150 \mathrm{My}$ of plant genome evolution

20

21

Our similarity searches and molecular biology approaches revealed patchy

22 conservation of Copia25. They show high sequence identity between a monocot genus of the

23 Musaceae family and two different dicotyledonous families in Asteridae: the Rubiaceae and

24 Solanaceae families. While monocot and dicot species diverged about $150 \mathrm{Mya}$, the Asteridae

25 and Rosidae lineages diverged $\sim 114$ Mya. More recently, Rubiaceae and Solanaceae diverged 
1 from their common ancestor about 83 Mya (Chaw et al. 2004; Wikstrom et al. 2001). This

2 discontinuous and incongruent distribution in dicots and monocots highlights a complex

3 evolutionary history of Copia25 in plants that could be traced back to the origin of 4 angiosperms.

Copia25-Coffea clusters in a strongly supported clade (100\% bootstrap value) with

6 homologous sequences from three Solanaceae species, S. tuberosum, N. tabacum and $N$.

7 benthamiana, and Musaceae species, Musa spp.. However, the nucleotide identity between

8 Copia25-Coffea and Copia25-Musa is higher than the one observed between Coffea and

9 potato and tobacco, and even in the comparison between Musa and Solanaceae (S. tuberosum:

$1077.4 \%$; N. benthamiana: 77.2\%). When the seven orthologous (COSII) genes showing the

11 highest sequence conservation are compared among the same species, the nucleotide identity

12 between C.canephora and M. balbisiana ranged from $67.8 \%$ to $80.2 \%$, less than the Copia 25

13 polyprotein identity for the same species comparison (Subfamily 1: 84.5\% and Subfamily 2:

$1485.5 \%$ ). Equivalent identities were also found in the gag region. Such outstandingly high

15 conservation raises questions about the molecular mechanisms, which are at its origin. Conservation of TEs between distantly related genera could be the result of different

17 and non-exclusive processes (Capy et al. 1994; Cummings 1994; Schaack et al. 2010; Wallau et al. 2011) such as: (i) domestication, (ii) conservation of functional sites, (iii) similarity of evolutionary rates, (iv) purifying selection and (v) horizontal transfer. The first two scenarios cannot explain the conservation of Copia25 across genera, since only portions of the TE are

21 generally domesticated and because the mechanisms of conserving functional sites 22 exclusively involve coding regions. High sequence identity was found for the full-length sequences of Copia25, including non-coding LTR regions. Similar TE evolutionary rate in

24 distinct species is an attractive hypothesis to explain the conservation observed in Copia25.

25 However, the TE evolutionary rate depends on multiple parameters such as the specific TE 
1 activity and the efficiency of TE host control mechanisms. Such a scenario remains unlikely

2 since these evolutionary mechanisms should be identical in several distantly-related species.

3 The fourth process, a purifying selection, would explain the high identity of a given TE

4 between distantly related species. The $\mathrm{Ka} / \mathrm{Ks}$ ratio estimated for pairwise comparisons of

5 Copia25 between Musa and Coffea sequences is low $(<0.3)$, denoting purifying selection and

6 explaining the conservation and the activity (at least until very recently) of this particular

7 element. However, the Ks values between Coffea and Solanaceae, Musa and Solanaceae and

$8 \quad$ Musa and Coffea species are at least twice as low for Copia25 as for COSII sequences. This

9 observation suggests that other evolutionary processes besides purifying selection might be

10 involved in Copia25 conservation. Finally, HTs of TEs, an occurrence suggested but rarely

11 confirmed in plants (Diao et al. 2006; El Baidouri et al. 2014; Fortune et al. 2008) may

12 explain the strong conservation level in coding and non-coding regions, and the sparse

13 distribution of TEs. However, HT scenarios first require ecological, chronological, and

14 geographical distribution overlapping between the species involved in the potential transfer to

15 be seriously considered. These requirements are not expected for Musa and Coffea, but a

16 chronological and geographical distribution overlap might have existed for the Musa and

17 Ixora species. The Ixora genus belongs to the Ixoroideae subfamily of the Rubiaceae family

18 such as the Coffea genus, but both belong to different tribes, Ixoreae and Coffeeae (Fig. S1).

19 The genus Musa evolved and diversified in tropical Asia (Liu et al. 2010), and the Musa

20 lineage ancestor originated $\sim 50$ Mya (Christelova et al. 2011). Likewise, the Ixora genus

21 originated in South-East Asia, in Borneo in particular (Lorence et al. 2007), and its ancestral

22 lineage originated 30 to 50 Mya (Tosh et al. 2013). Therefore, the ancestors of Musa and

23 Ixora could have shared the same period and geographical origin. The hypothesis of the HT of

24 Copia25 between the ancestors of Ixora and Musa is therefore supported by the chronological

25 and geographical distribution of species. This hypothesis is also supported by the high global 
1 sequence identity as well as by the $K s$ values, which are much lower for Copia 25 than for the

2 COSII, suggesting that its presence is recent in the Musa genome. Furthermore, the phylogeny

3 of Copia25 RT including the Musa and Rubiaceae species sequences clearly indicates a

4 strong relationship between Copia25-Musa and Copia25-Ixora (Fig. 4). This relationship does

5 not result from similar selective pressure acting in both groups (as showed by LRT analyses,

6 which exclude purifying selection as the process responsible for sequence similarity) and thus

7 reinforces the proposition of HT. The putative period of Copia25 transfer from Ixora to Musa

8 can be estimated by the molecular clock equation using the RT sequences ( $375 \mathrm{nt} ; K s$ ranged

9 from 0.25 to 0.56 ). The estimated age range from 19 to 43 Mya is congruent with the period

10 when the ancestors of both genera shared geographical distribution. This estimation must be

11 considered with caution because of the short sequence used for establishing the time of

12 divergence and because the molecular clock used is not calibrated for Rubiaceae. Our results

13 thus suggest a potential and ancestral HT of Copia25 from Ixora to Musa (Fig. S8).

With the facility for plants to inter-cross and given the autonomy of their germ line, plant genomes have a natural propensity to transfer genetic material. They also have a high content of LTR-RTs, elements whose cytoplasmic multiplication phase heightens the

17 likelihood of being captured and exchanged among other species, thus favoring potential HT.

18 Thanks to the fast-growing number of data sequences available, more studies are being 19 conducted involving several species. Their results reveal scenarios of complex evolution, 20 particularly those concerning TEs. Here, our detailed analyses of Copia25 in angiosperms 21 disclose the complexity of the evolutionary dynamics of this ancient element, involving 22 several processes including sequence conservation, rapid turnover, stochastic losses and 23 horizontal transfer. Additional information on the presence and the activity of Copia25 in 24 angiosperms is required to precisely identify the mechanism involved in such remarkable 
1 conservation of a transposable element harbored by large and divergent groups of plant

2 species.

3

4

5 Acknowledgments

6

7 This research was supported Agropolis Fondation through the "Investissement d'avenir" 8 program (ANR-10-LABX-0001-01) under the reference ID 1002-009 and 1102-006, CAPES

9 (Grants 01/2010 to CMAC and fellowship 9127-11-9 to ESD), Brazilian agencies FAPESP

10 (Fundação de Amparo à Pesquisa do Estado de São Paulo - Grant 2013/15070-4 to CMAC

11 and fellowship 2011/18226-0 to ESD) and CNPq (Conselho Nacional de Desenvolvimento

12 Científico e Tecnológico - Grant 306493/2013-6 to CMAC) and French agency ANR (Agence

13 Nationale de la Recherche; Genoplante ANR-08- GENM-022-001). Acknowledgments to Dr.

14 A. D'Hont for providing Musa spp. DNA samples; Herman E. Taedoumg for providing

15 Craterispermum samples; Dr. P. De Block for providing Rubiaceae samples; Dr. J-J.

16 Rakotomalala for providing Mascarocoffea samples. Acknowledgements to Philippe

17 Lashermes and the Coffee Genome Consortium for the availability of the $C$. canephora BAC-

18 end sequences and draft genome.

19

20

21 Conflict of Interest The authors declare that they have no competing interests.

22

23

24 Electronic supplementary material

25 The paper contains supplementary material, File 1. 


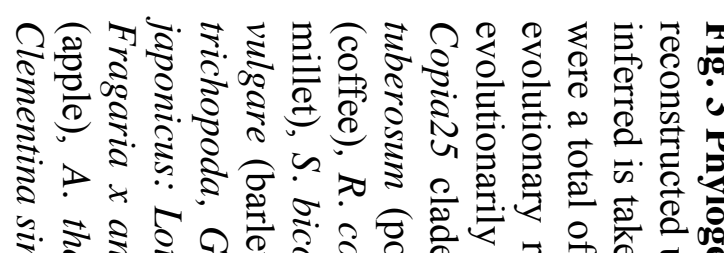

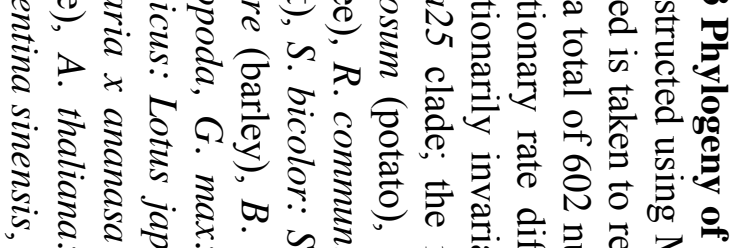

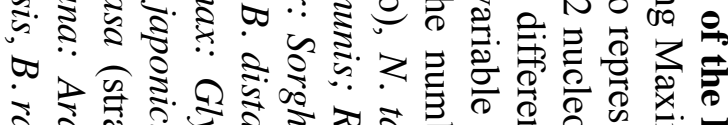

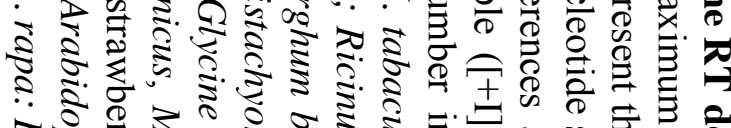

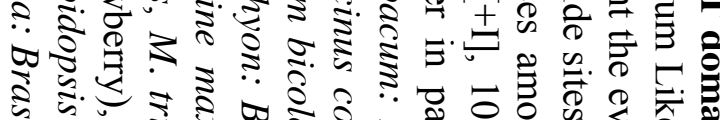

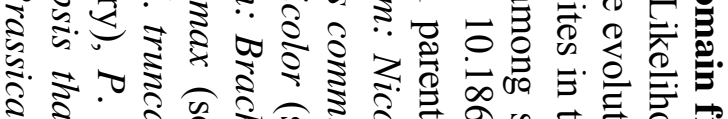

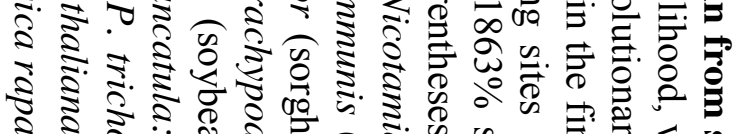
等

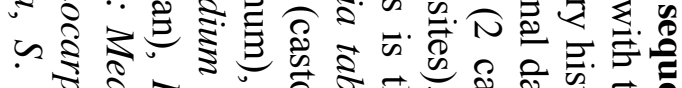

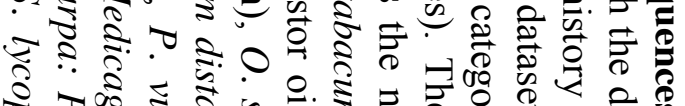

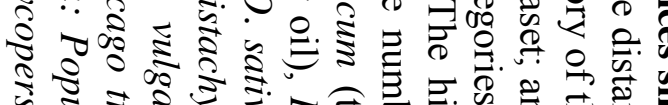

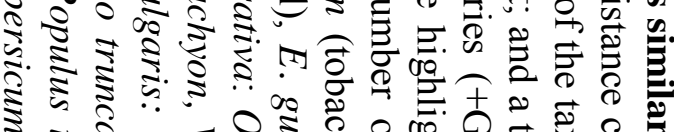

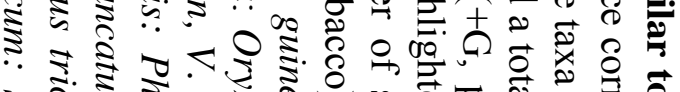

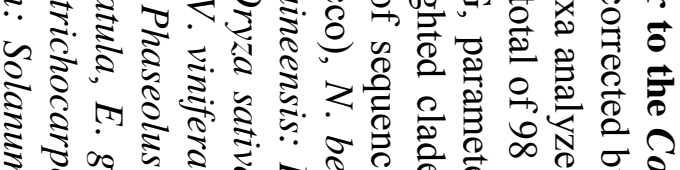
章 o

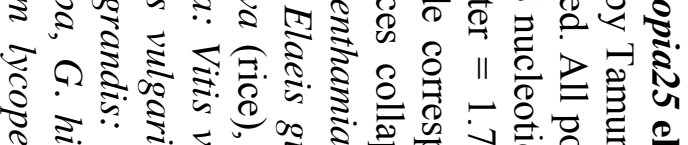

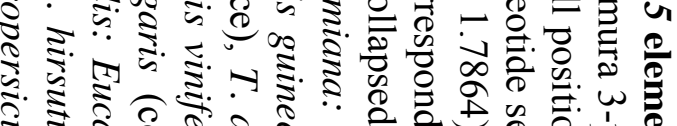

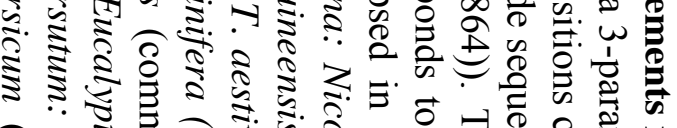

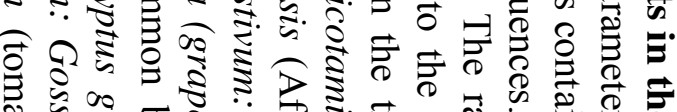

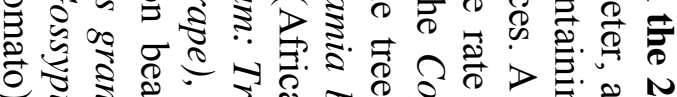

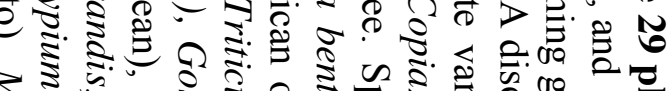

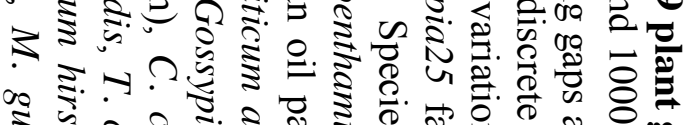

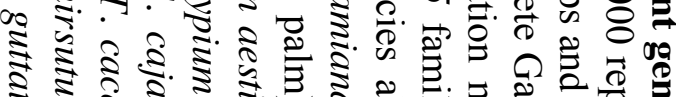

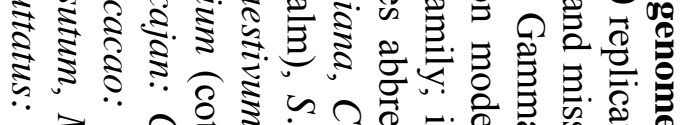

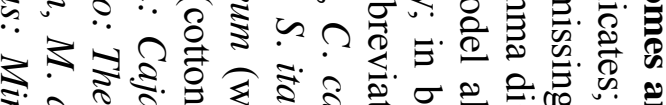

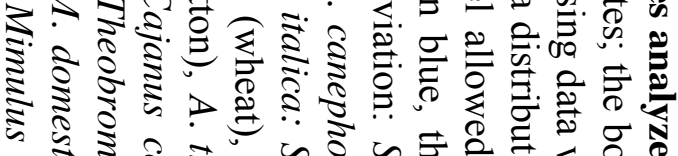

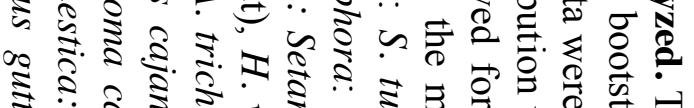

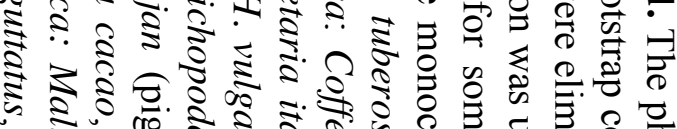

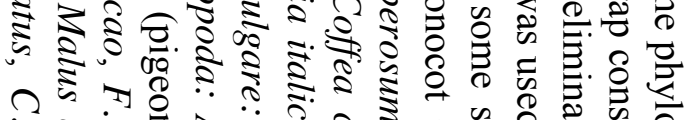

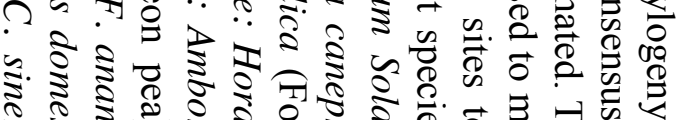



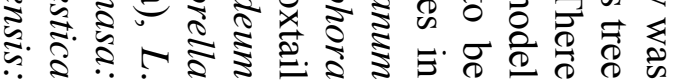

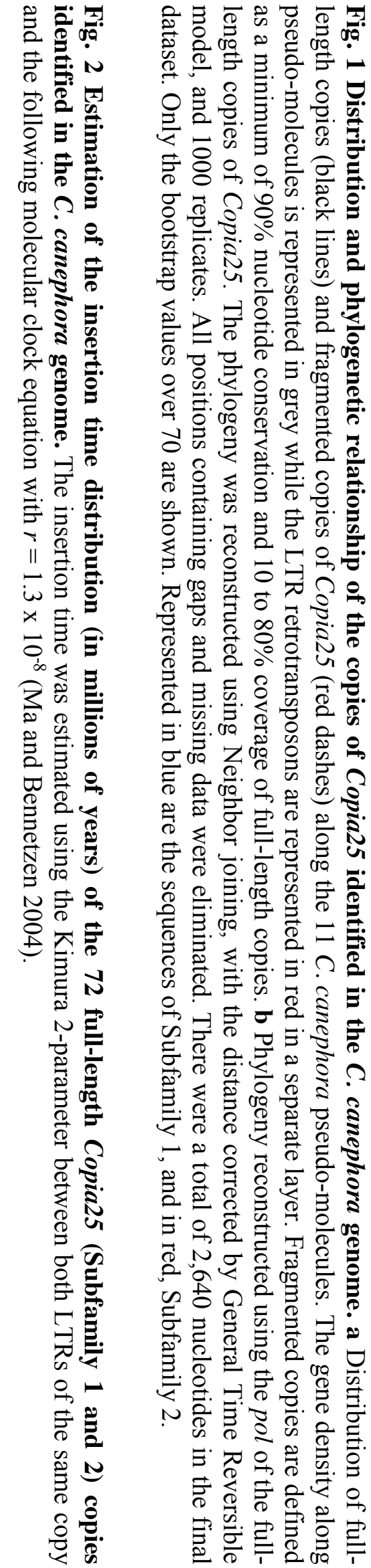

की



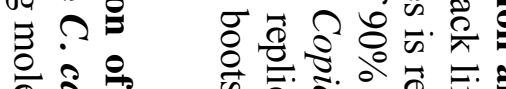

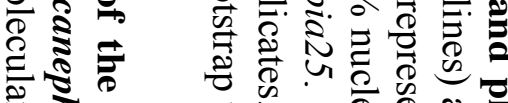

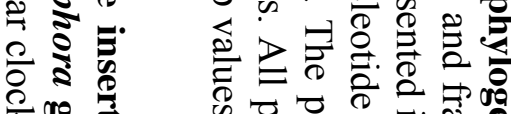
\&

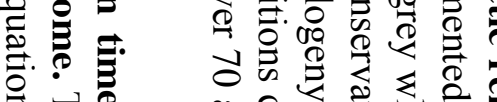

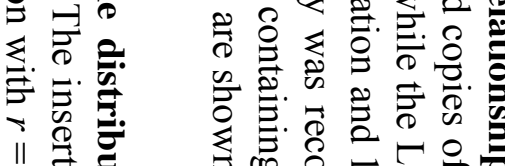

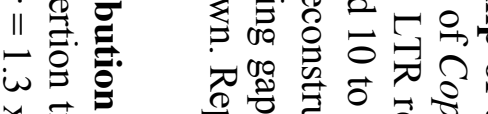

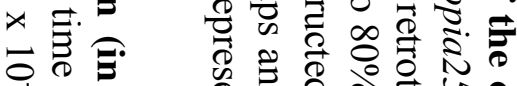

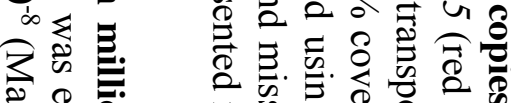

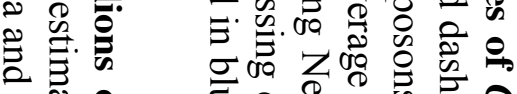

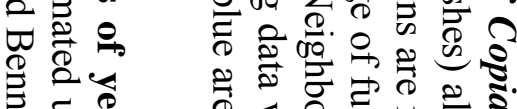

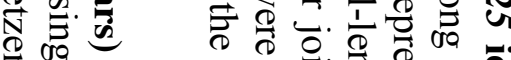

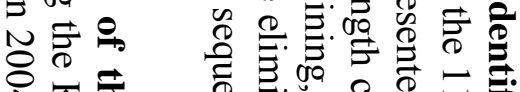
页言

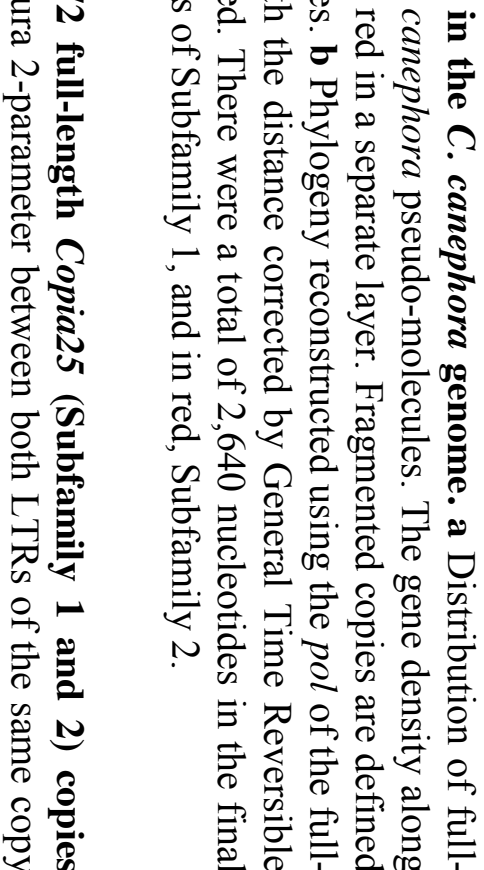




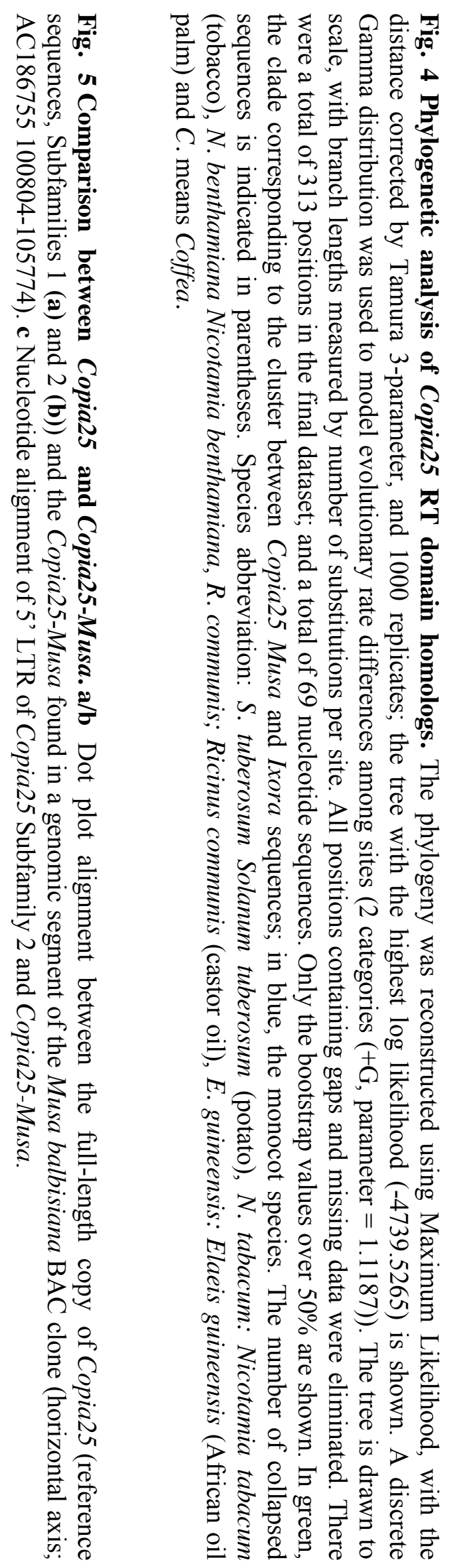




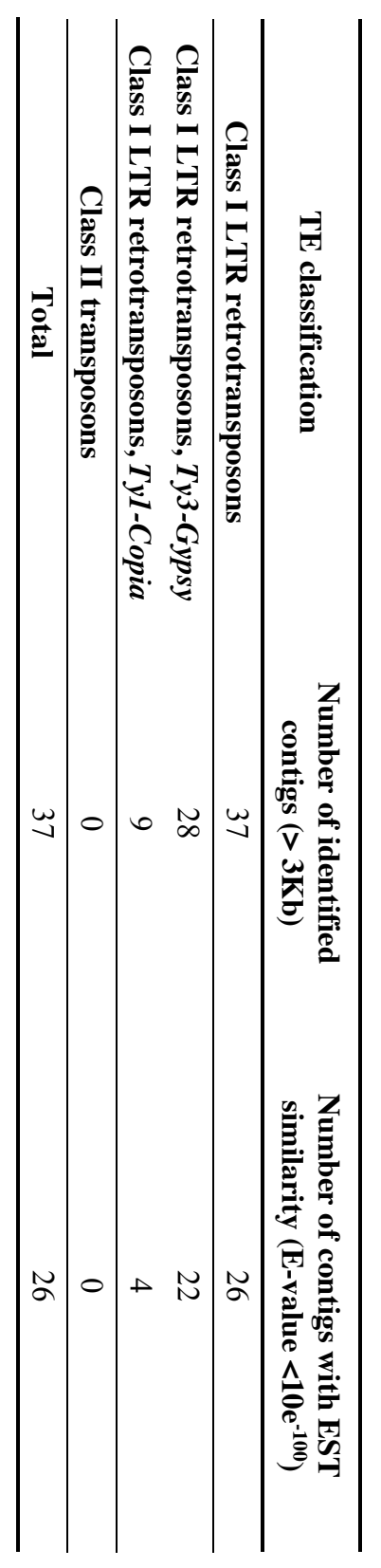

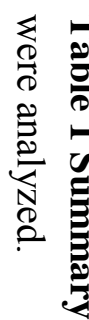

疍

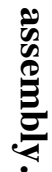

きٍ

$\stackrel{8}{\stackrel{\Xi}{90}}$

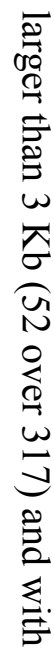

2
8
0
0
0

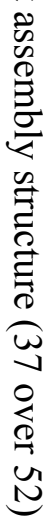




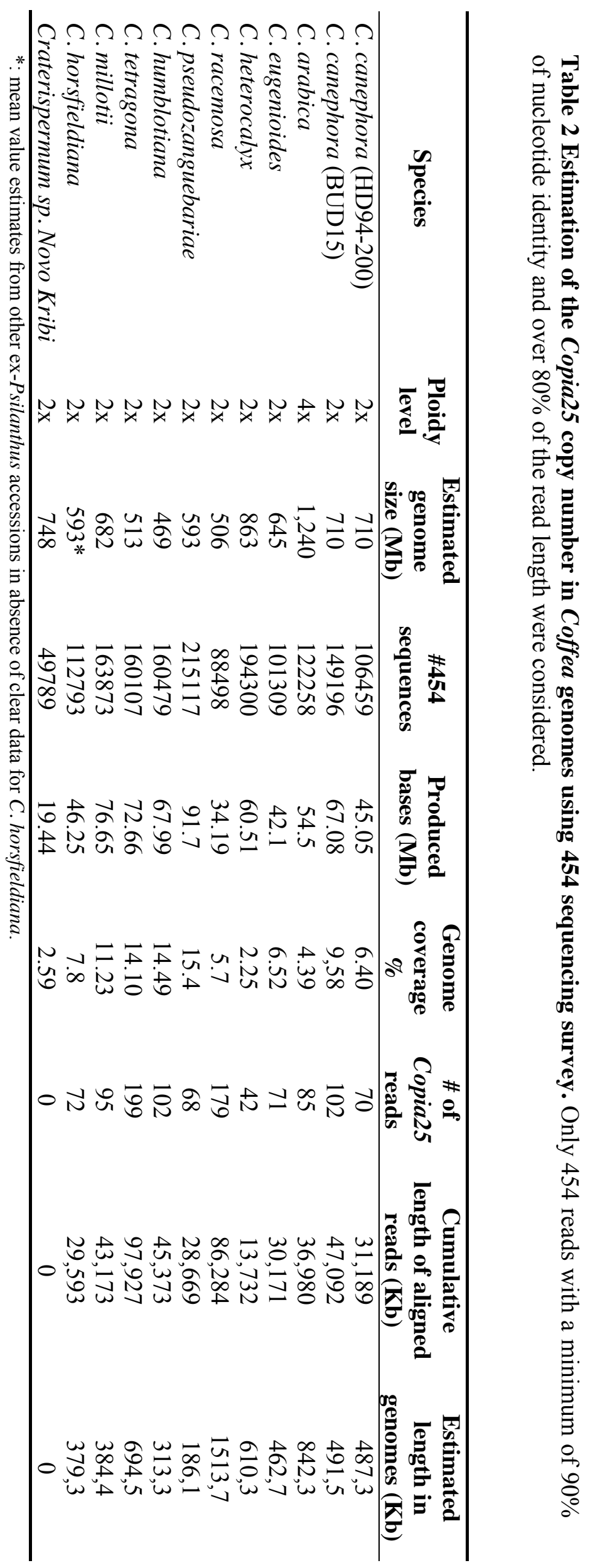


Table 3 Likelihood ratio test for testing models of sequence evolution for Copia25 retrotransposons.

\begin{tabular}{|c|c|c|c|c|c|c|c|}
\hline Model & & Parameter & $\ell$ & $2 \Delta \ell$ & $\omega_{\mathrm{B}}$ & $\omega_{\mathrm{F}}$ & Conclusion \\
\hline \multirow[t]{2}{*}{ One-ratio } & Model I & $\omega$ free & -2469.160 & \multirow{2}{*}{$239.308^{* *}$} & 0.191 & - & \multirow{2}{*}{ Purifying selection in the Copia 25 tree } \\
\hline & Model II & $\omega=1$ & -2588.814 & & - & - & \\
\hline \multirow{2}{*}{ Two-ratio } & Model IV & $\omega=1$ & -2485.246 & $33.568 * *$ & 0.198 & 1 & Purifying selection in the Ixora Copia 25 clade \\
\hline & Model V & $\omega$ free & -2463.734 & 2.526 & 0.169 & 0.552 & Neutral evolution in the Musa Copia 25 clade \\
\hline
\end{tabular}

Critical values of $\mathrm{X}^{2}, 1 \mathrm{df}{ }^{*}: 3.84 ; * * 6.63 ; 2 \Delta \ell=2\left(1_{1}-1_{0}\right)$ 

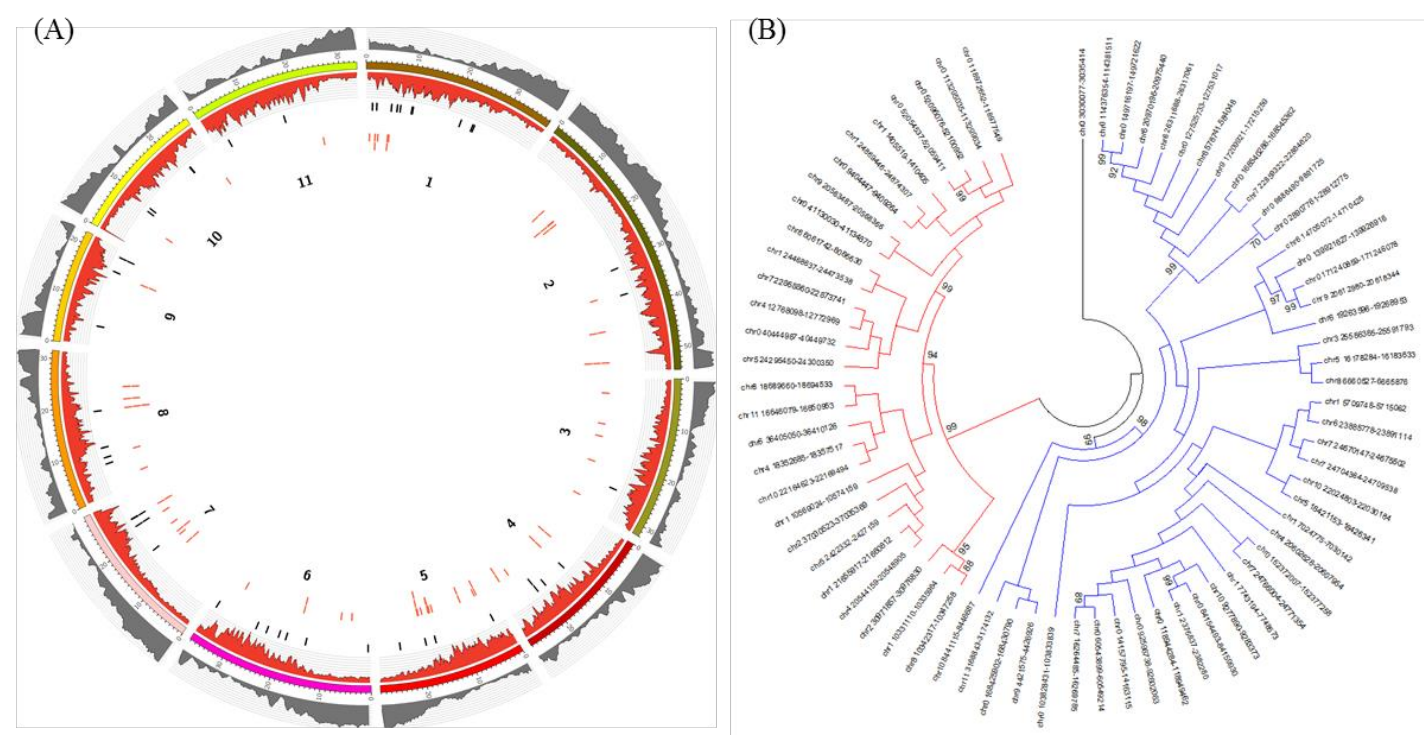

Fig. 1 Distribution and phylogenetic relationship of the copies of Copia25 identified in the $\boldsymbol{C}$. canephora genome. a Distribution of full-length copies (black lines) and fragmented copies of Copia25 (red dashes) along the 11 C. canephora pseudomolecules. The gene density along pseudo-molecules is represented in grey while the LTR retrotransposons are represented in red in a separate layer. Fragmented copies are defined as a minimum of $90 \%$ nucleotide conservation and 10 to $80 \%$ coverage of fulllength copies. b Phylogeny reconstructed using the pol of the full-length copies of Copia25. The phylogeny was reconstructed using Neighbor joining, with the distance corrected by General Time Reversible model, and 1000 replicates. All positions containing gaps and missing data were eliminated. There were a total of 2,640 nucleotides in the final dataset. Only the bootstrap values over 70 are shown. Represented in blue are the sequences of Subfamily 1, and in red, Subfamily 2. 

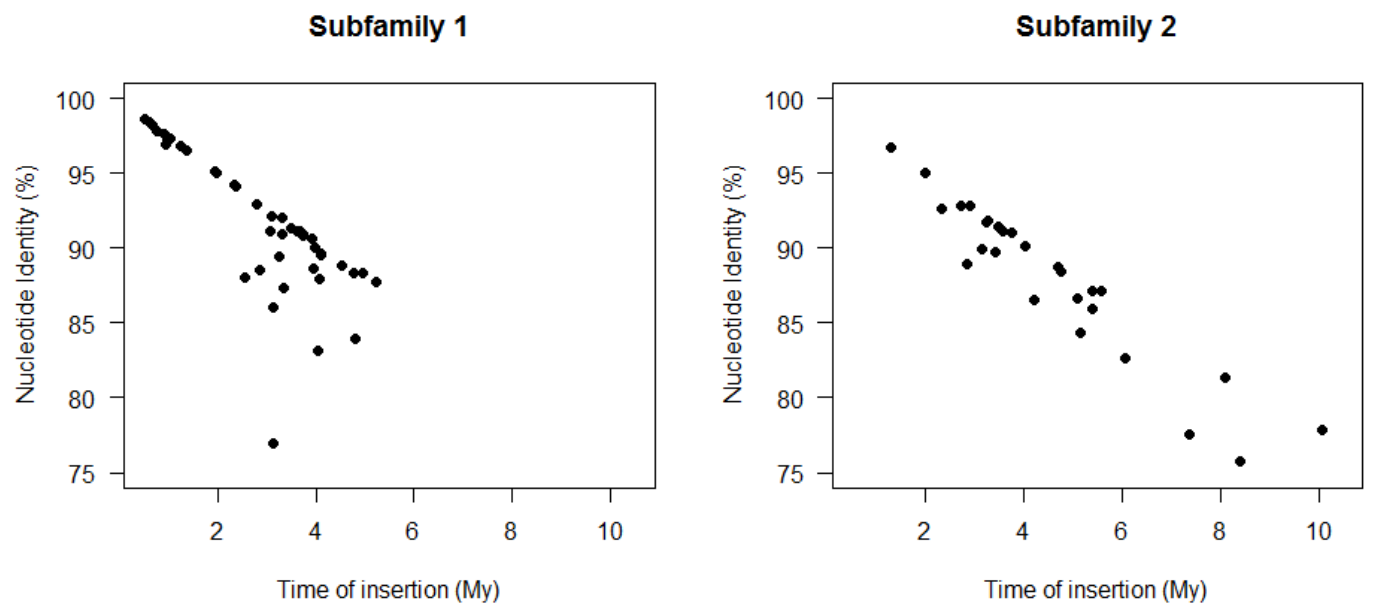

Fig. 2 Estimation of the insertion time distribution (in millions of years) of the 72 full-length Copia25 (Subfamily 1 and 2) copies identified in the $C$. canephora genome. The insertion time was estimated using the Kimura 2-parameter between both LTRs of the same copy and the following molecular clock equation with $r=1.3 \times 10^{-8}$ (Ma and Bennetzen 2004). 


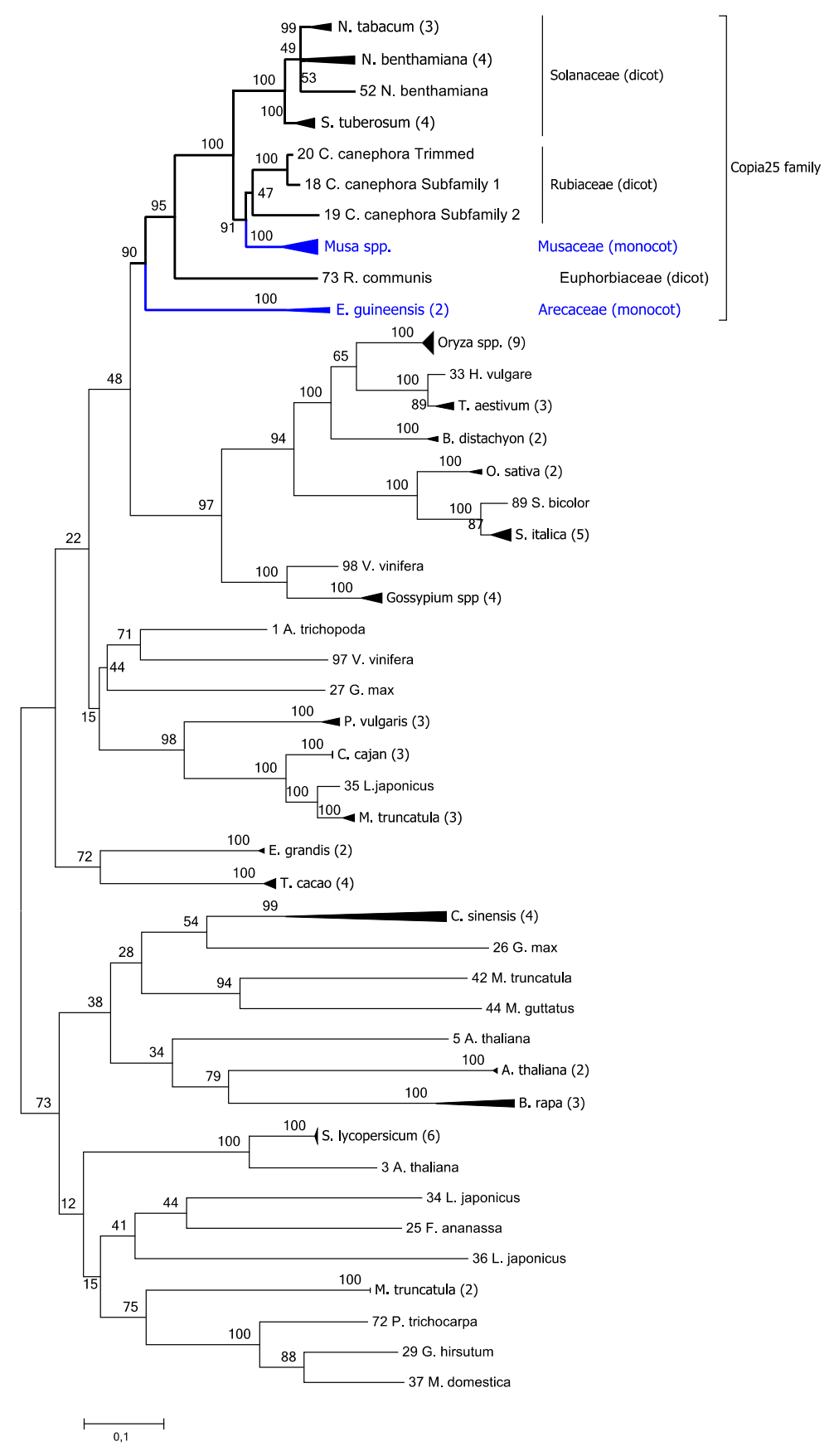

Fig. 3 Phylogeny of the RT domain from sequences similar to the Copia25 elements in the 29 plant genomes analyzed. The phylogeny was reconstructed using Maximum Likelihood, with the distance corrected by Tamura 3-parameter, and 1000 replicates; the bootstrap consensus tree inferred is taken to represent the evolutionary history of the taxa analyzed. All positions containing gaps and missing data were eliminated. There were a total of 602 nucleotide sites in the final dataset; and a total of 98 nucleotide sequences. A discrete Gamma distribution was used to model evolutionary rate differences among sites $(2$ categories $(+\mathrm{G}$, parameter $=1.7864))$. The rate variation model allowed for some sites to be evolutionarily invariable $([+\mathrm{I}], 10.1863 \%$ sites $)$. The highlighted clade corresponds to the Copia25 family; in blue, the monocot species in Copia 25 clade; the number in parentheses is the number of sequences collapsed in the 
tree. Species abbreviation: S. tuberosum Solanum tuberosum (potato), N. tabacum: Nicotamia tabacum (tobacco), N. benthamiana: Nicotamia benthamiana, C. canephora: Coffea canephora (coffee), R. communis; Ricinus communis (castor oil), E. guineensis: Elaeis guineensis (African oil palm), S. italica: Setaria italica (Foxtail millet), S. bicolor: Sorghum bicolor (sorghum), O. sativa: Oryza sativa (rice), T. aestivum: Triticum aestivum (wheat), H. vulgare: Hordeum vulgare (barley), B. distachyon: Brachypodium distachyon, V. vinifera: Vitis vinifera (grape), Gossypium (cotton), A. trichopoda: Amborella trichopoda, G. max: Glycine max (soybean), P. vulgaris: Phaseolus vulgaris (common bean), C. cajan: Cajanus cajan (pigeon pea), L. japonicus: Lotus japonicus, M. truncatula: Medicago truncatula, E. grandis: Eucalyptus grandis, T. cacao: Theobroma cacao, F. ananasa: Fragaria x ananasa (strawberry), P. trichocarpa: Populus trichocarpa, G. hirsutum: Gossypium hirsutum, M. domestica: Malus domestica (apple), A. thaliana: Arabidopsis thaliana, S. lycopersicum: Solanum lycopersicum (tomato), M. guttatus: Mimulus guttatus, C. sinensis: Clementina sinensis, B. rapa: Brassica rapa. 


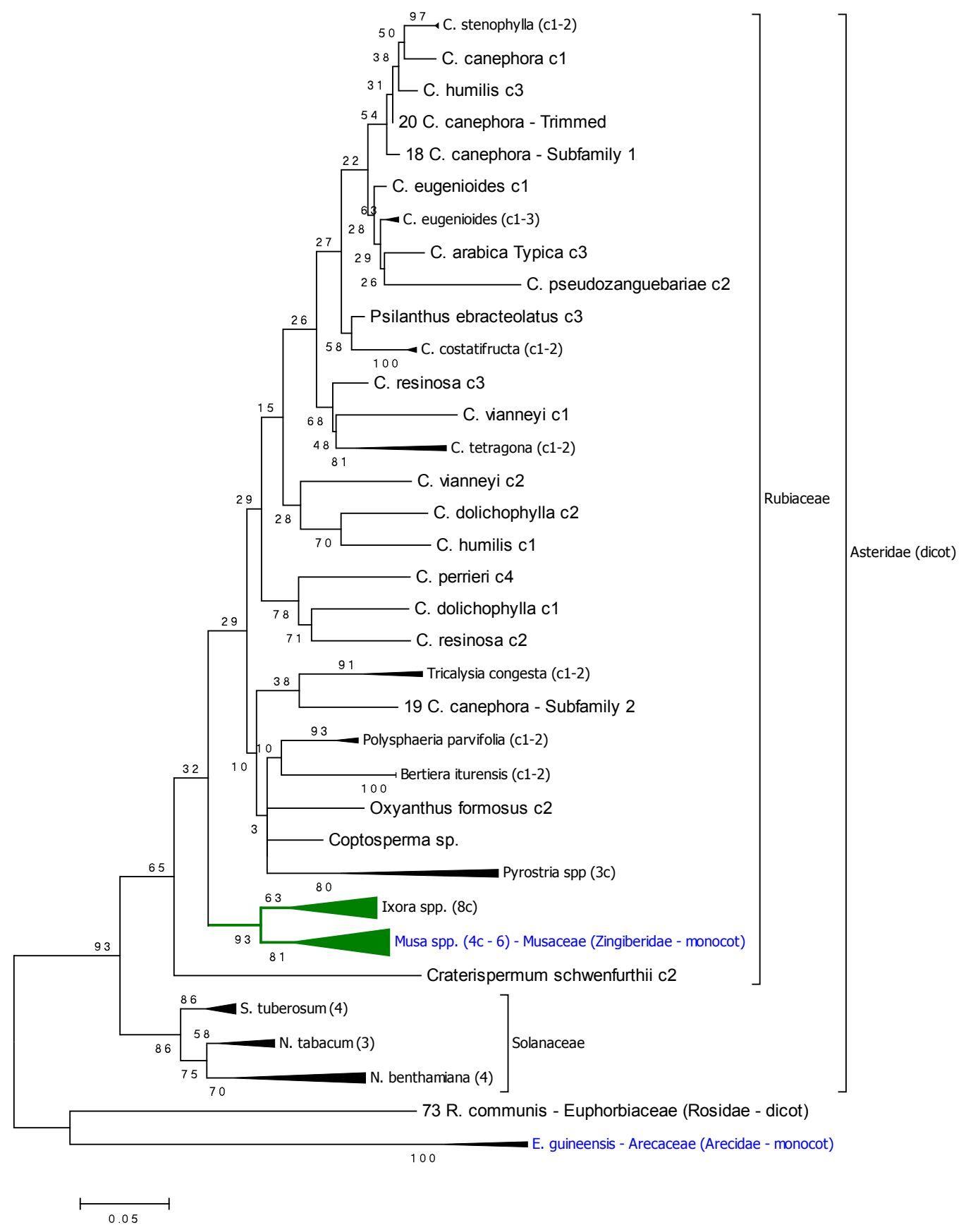

Fig. 4 Phylogenetic analysis of Copia25 RT domain homologs. The phylogeny was reconstructed using Maximum Likelihood, with the distance corrected by Tamura 3parameter, and 1000 replicates; the tree with the highest log likelihood (-4739.5265) is shown. A discrete Gamma distribution was used to model evolutionary rate differences among sites $(2$ categories $(+\mathrm{G}$, parameter $=1.1187))$. The tree is drawn to scale, with branch lengths measured by number of substitutions per site. All positions containing gaps and missing data were eliminated. There were a total of 313 positions in the final dataset; and a total of 69 nucleotide sequences. Only the bootstrap values over $50 \%$ are shown. In green, the clade corresponding to the cluster between Copia25 Musa and Ixora sequences; in blue, the monocot species. The number of collapsed sequences is 
indicated in parentheses. Species abbreviation: S. tuberosum Solanum tuberosum (potato), N. tabacum: Nicotamia tabacum (tobacco), N. benthamiana Nicotamia benthamiana, R. communis; Ricinus communis (castor oil), E. guineensis: Elaeis guineensis (African oil palm) and C. means Coffea. 
(A)

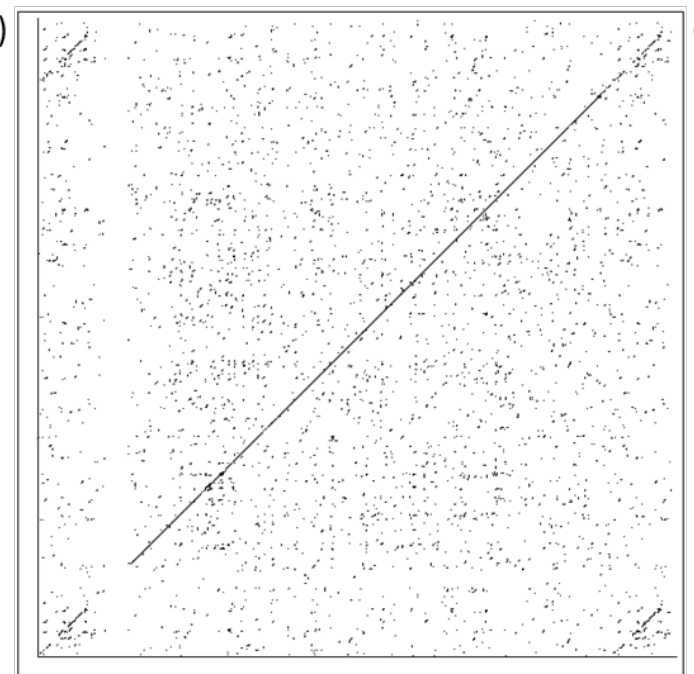

(B)

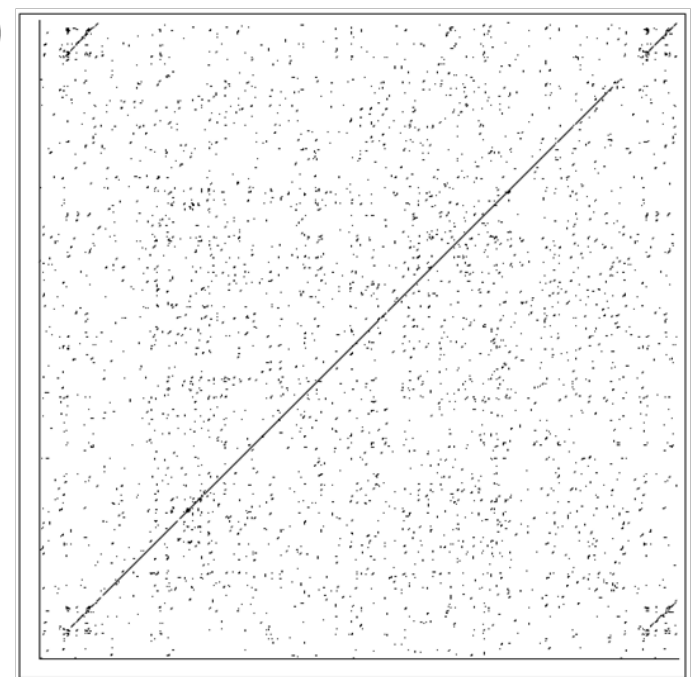

(C)

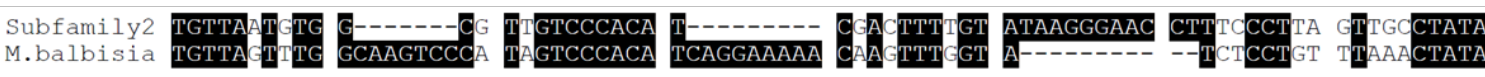

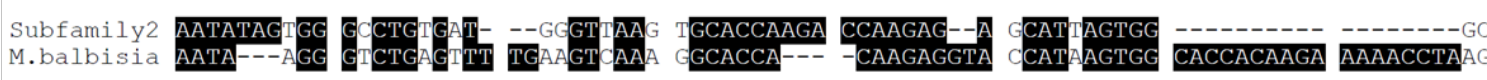

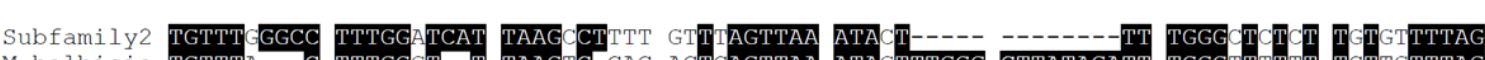

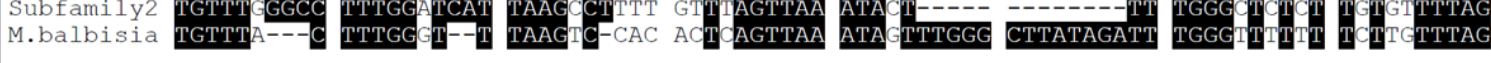



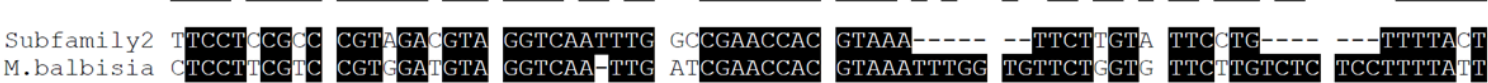

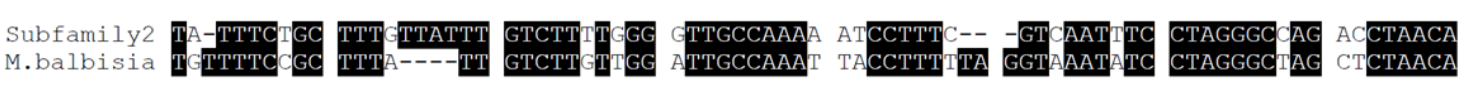

Fig. 5 Comparison between Copia25 and Copia25-Musa. Dot plot alignment between the full-length copy of Copia25 (reference sequences, a Subfamilies 1 and b 2) and the Copia25-Musa found in a genomic segment of the Musa balbisiana BAC clone (horizontal axis; AC186755 100804-105774). c Nucleotide alignment of 5' LTR of Copia25 Subfamily 2 and Copia25-Musa. 


\section{References}

Anisimova M, Ziheng Y (2007) Multiple Hypothesis Testing to Detect Lineages under Positive Selection that Affects Only a Few Sites. Mol Biol Evol 24:1219-1228

Capy P, Anxolabehere D, Langin T (1994) The strange phylogenies of transposable elements: are horizontal transfers the only explantation? Trends Genet 10:7-12

Carver TJ, Rutherford KM, Berriman M, Rajandream MA, Barrell BG, Parkhill J (2005) ACT: the Artemis Comparison Tool. Bioinformatics 21:3422-3423

Chaw SM, Chang CC, Chen HL, Li WH (2004) Dating the monocot-dicot divergence and the origin of core eudicots using whole chloroplast genomes. J Mol Evol $58: 424-441$

Cheng X, Zhang D, Cheng Z, Keller B, Ling HQ (2009) A new family of Ty1-copialike retrotransposons originated in the tomato genome by a recent horizontal transfer event. Genetics 181:1183-1193

Chevalier A (1946) Ecologie et distribution géographique des caféiers sauvages et cultivés. Rev Bot Appl Agric Trop 26:81-94

Christelova P, Valarik M, Hribova E, De Langhe E, Dolezel J (2011) A multi gene sequence-based phylogeny of the Musaceae (banana) family. BMC Evol Biol $11: 103$

Cummings MP (1994) Transmission patterns of eukaryotic transposable elements: arguments for and against horizontal transfer. Trends Ecol Evol 9:141-145

D'Hont A, Denoeud F, Aury J-M, et al (2012) The banana (Musa acuminata) genome and the evolution of monocotyledonous plants. Nature 488:213-217

Davis AP (2010) Six species of Psilanthus transferred to Coffea (Coffeeae, Rubiaceae). Phytotaxa 10:41-45

Davis AP (2011) Psilanthus mannii, the type species of Psilanthus, transferred to Coffea. Nordic Journal of Botany 29:471-472

de Carvalho MO, Loreto EL (2012) Methods for detection of horizontal transfer of transposable elements in complete genomes. Genetics and molecular biology 35:1078-1084

DeBarry JD, Liu R, Bennetzen JL (2008) Discovery and assembly of repeat family pseudomolecules from sparse genomic sequence data using the Assisted Automated Assembler of Repeat Families (AAARF) algorithm. BMC Bioinformatics 9:235

Denoeud F, Carretero-Paulet L, Dereeper A, et al (2014) The coffee genome provides insight into the convergent evolution of caffeine biosynthesis. Science 345:11814 
Dereeper A, Guyot R, Tranchant-Dubreuil C, et al (2013) BAC-end sequences analysis provides first insights into coffee (Coffea canephora $\mathrm{P}$.) genome composition and evolution. Plant Mol Biol 83:177-89

Diao X, Freeling M, Lisch D (2006) Horizontal transfer of a plant transposon. PLoS Biol 4:e5

El Baidouri M, Carpentier M-CC, Cooke R, et al (2014) Widespread and frequent horizontal transfers of transposable elements in plants. Genome Res 24:831-8

Ellinghaus D, Kurtz S, Willhoeft U (2008) LTRharvest, an efficient and flexible software for de novo detection of LTR retrotransposons. BMC Bioinformatics 9:18

Fortune PM, Roulin A, Panaud O (2008) Horizontal transfer of transposable elements in plants. Commun Integr Biol 1:74-77

Gaut BS, Morton BR, McCaig BC, Clegg MT (1996) Substitution rate comparisons between grasses and palms: synonymous rate differences at the nuclear gene Adh parallel rate differences at the plastid gene rbcL. Proc Natl Acad Sci U S A 93:10274-10279

Hamon P, Duroy P-OO, Dubreuil-Tranchant C, et al (2011) Two novel Ty1-copia retrotransposons isolated from coffee trees can effectively reveal evolutionary relationships in the Coffea genus (Rubiaceae). Mol Genet Genomics 285:447-60

Jiang N, Gao D, Xiao H, van der Knaap E (2009) Genome organization of the tomato sun locus and characterization of the unusual retrotransposon Rider. Plant $\mathrm{J}$ 60:181-193

Jiang N, Visa S, Wu S, van der Knaap E (2012) Rider Transposon Insertion and Phenotypic Change in Tomato. Topics in Current Genetics 24:297-312

Jurka J, Kapitonov VV, Pavlicek A, Klonowski P, Kohany O, Walichiewicz J (2005) Repbase Update, a database of eukaryotic repetitive elements. Cytogenet Genome Res 110:462-467

Kumar S, Nei M, Dudley J, Tamura K (2008) MEGA: a biologist-centric software for evolutionary analysis of DNA and protein sequences. Brief Bioinform 9:299-306

Lashermes P, Combes MC, Robert J, Trouslot P, D'Hont A, Anthony F, Charrier A (1999) Molecular characterisation and origin of the Coffea arabica L. genome. Mol Gen Genet 261:259-266

Lescot M, Piffanelli P, Ciampi A, et al (2008) Insights into the Musa genome: Syntenic relationships to rice and between Musa species. BMC Genomics 9:58.

Librado P, Rozas J (2009) DnaSP v5: a software for comprehensive analysis of DNA polymorphism data. Bioinformatics 25:1451-1452 
Liu A, Kress W, Li D (2010) Phylogenetic analyses of the banana family (Musaceae) based on nuclear ribosomal (ITS) and chloroplast (trnL-F) evidence. Taxon 59:20-28

Llorens C, Futami R, Covelli L, et al. (2010) The Gypsy Database (GyDB) of mobile genetic elements: release 2.0. Nucleic Acids Res 39:D70-74

Lorence D, Wagner W, Mouly A, Florence J (2007) Revision of Ixora (Rubiaceae) in the Marquesas Islands (French Polynesia). Botanical Journal of The Linnean Society 155:581-597

Ma J, Bennetzen J (2004) Rapid recent growth and divergence of rice nuclear genomes. Proceedings of the National Academy of Sciences of the United States of America 101:12404-12410

Marraccini P, Freire LP, Alves GS, et al (2011) RBCS1 expression in coffee: Coffea orthologs, Coffea arabica homeologs, and expression variability between genotypes and under drought stress. BMC Plant Biol 11:85

Maurin O, Davis AP, Chester M, Mvungi EF, Jaufeerally-Fakim Y, Fay MF (2007) Towards a Phylogeny for Coffea (Rubiaceae): identifying well-supported lineages based on nuclear and plastid DNA sequences. Ann Bot (Lond) 100:1565-1583

Michael TP, Jackson S (2013) The First 50 Plant Genomes. The Plant Genome 6:1-7

Moisy C, Schulman AH, Kalendar R, et al (2014) The Tvv1 retrotransposon family is conserved between plant genomes separated by over 100 million years. Theor Appl Genet 127:1223-35

Moschetto D, Montagnon C, Guyot B, Perriot JJ, Leroy T, Eskes A (1996) Studies on the effect of genotype on cup quality of Coffea canephora. Tropical Science $36: 18-31$

Roulin A, Piegu B, Wing RA, Panaud O (2008) Evidence of multiple horizontal transfers of the long terminal repeat retrotransposon RIRE1 within the genus Oryza. Plant J 53:950-959

SanMiguel P, Gaut BS, Tikhonov A, Nakajima Y, Bennetzen JL (1998) The paleontology of intergene retrotransposons of maize. Nat Genet 20:43-45

SanMiguel P, Tikhonov A, Jin YK, et al. (1996) Nested retrotransposons in the intergenic regions of the maize genome. Science 274: 765-768.

Schaack S, Gilbert C, Feschotte C (2010) Promiscuous DNA: horizontal transfer of transposable elements and why it matters for eukaryotic evolution. Trends Ecol Evol 25:537-546 
Sonnhammer EL, Durbin R (1995) A dot-matrix program with dynamic threshold control suited for genomic DNA and protein sequence analysis. Gene 167:GC110

Tamura K, Stecher G, Peterson D, Filipski A (2013) MEGA6: molecular evolutionary genetics analysis version 6.0. Mol Biol Evol 30:2725-2729

Tosh J, Dessein S, Buerki S, et al (2013) Evolutionary history of the Afro-Madagascan Ixora species (Rubiaceae): species diversification and distribution of key morphological traits inferred from dated molecular phylogenetic trees. Annals of Botany 112:1723-1742

Vitte C, Panaud O, Quesneville H (2007) LTR retrotransposons in rice (Oryza sativa, L.): recent burst amplifications followed by rapid DNA loss. BMC Genomics $8: 218$

Wallau GL, Hua-Van A, Capy P, Loreto EL (2011) The evolutionary history of mariner-like elements in Neotropical drosophilids. Genetica 139:327-338

Wicker T, Keller B (2007) Genome-wide comparative analysis of copia retrotransposons in Triticeae, rice, and Arabidopsis reveals conserved ancient evolutionary lineages and distinct dynamics of individual copia families. Genome Res 17:1072-1081

Wicker T, Sabot F, Hua-Van A, et al (2007) A unified classification system for eukaryotic transposable elements. Nature Reviews Genetics 8:973-982

Wikstrom N, Savolainen V, Chase MW (2001) Evolution of the angiosperms: calibrating the family tree. Proc Biol Sci 268:2211-2220

Wu F, Mueller LA, Crouzillat D, Petiard V, Tanksley SD (2006) Combining bioinformatics and phylogenetics to identify large sets of single-copy orthologous genes (COSII) for comparative, evolutionary and systematic studies: a test case in the euasterid plant clade. Genetics 174:1407-1420

Xiao H, Jiang N, Schaffner E, Stockinger EJ, van der Knaap E (2008) A retrotransposon-mediated gene duplication underlies morphological variation of tomato fruit. Science 319:1527-1530

Yang Z (1997) PAML: a program package for phylogenetic analysis by maximum likelihood. Comput Appl Biosci 13:555-556

Yang Z, Nielsen R (1998) Synonymous and nonsynonymous rate variation in nuclear genes of mammals. J Mol Evol 46:409-418

Yu Q, Guyot R, Kochko A de, et al (2011) Micro-collinearity and genome evolution in the vicinity of an ethylene receptor gene of cultivated diploid and allotetraploid coffee species (Coffea). Plant J 67:305-17 
Yuyama PM, Pereira LF, Santos TB dos, et al (2012) FISH using a gag-like fragment probe reveals a common Ty3-gypsy-like retrotransposon in genome of Coffea species. Genome 55:825-33 

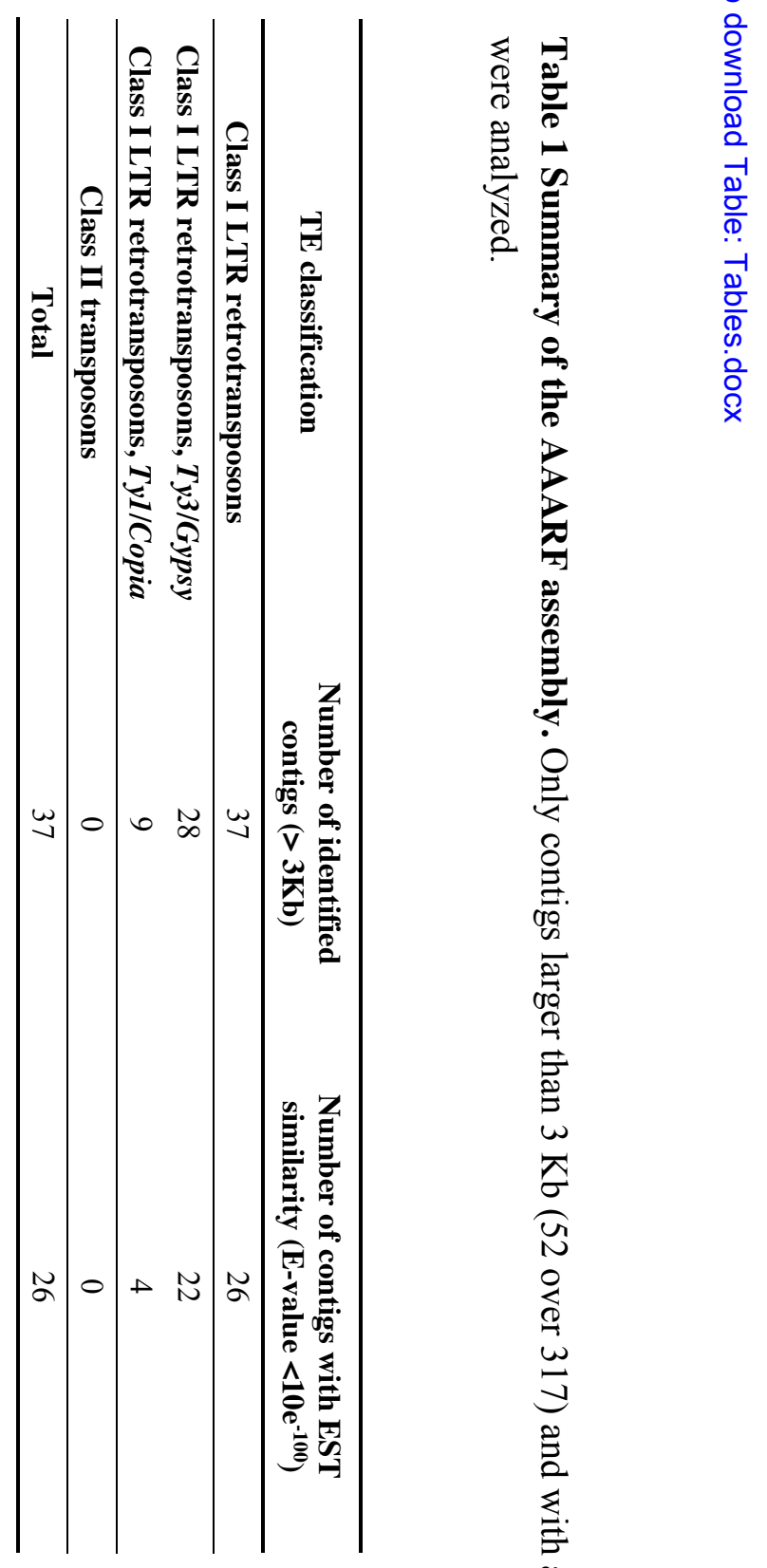


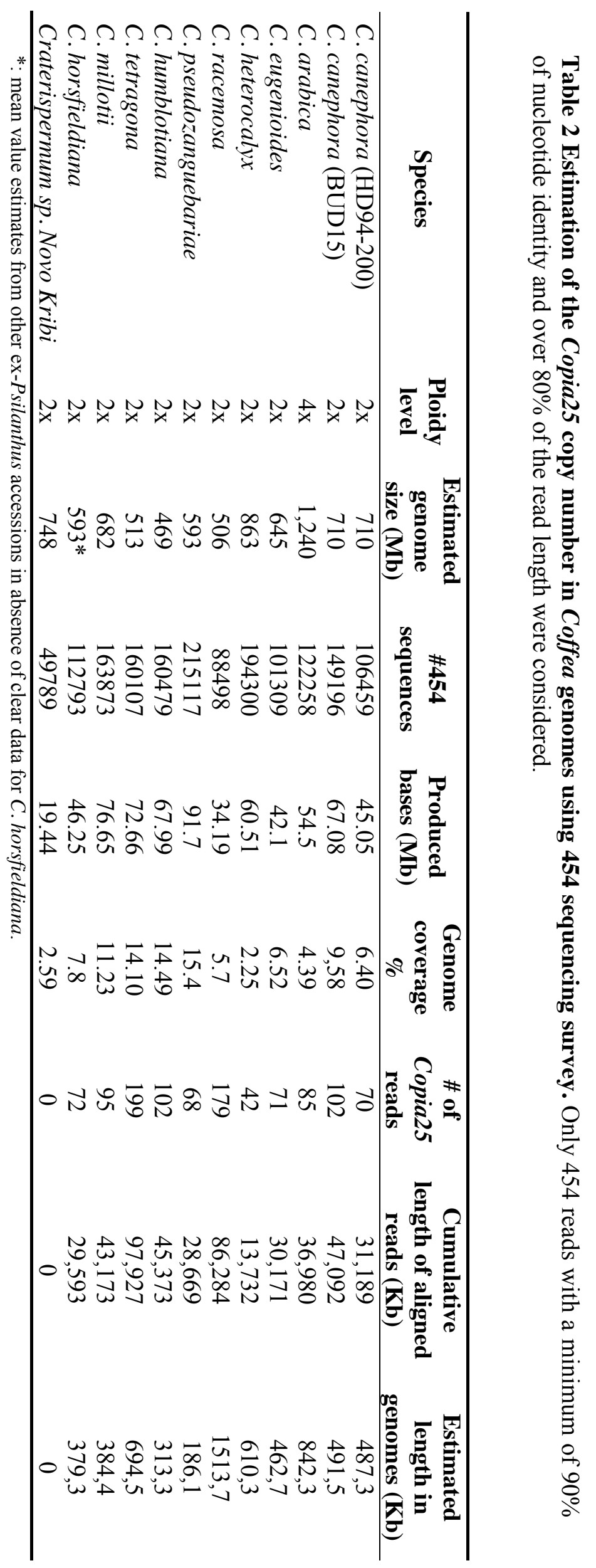


Table 3 Likelihood ratio test for testing models of sequence evolution for Copia25 retrotransposons.

\begin{tabular}{|c|c|c|c|c|c|c|c|}
\hline Model & & Parameter & $\ell$ & $2 \Delta \ell$ & $\omega_{\mathrm{B}}$ & $\omega_{\mathrm{F}}$ & Conclusion \\
\hline \multirow[t]{2}{*}{ One-ratio } & Model I & $\omega$ free & -2469.160 & \multirow{2}{*}{$239.308^{* *}$} & 0.191 & - & \multirow{2}{*}{ Purifying selection in the Copia 25 tree } \\
\hline & Model II & $\omega=1$ & -2588.814 & & - & - & \\
\hline \multirow{2}{*}{ Two-ratio } & Model IV & $\omega=1$ & -2485.246 & $33.568 * *$ & 0.198 & 1 & Purifying selection in the Ixora Copia 25 clade \\
\hline & Model V & $\omega$ free & -2463.734 & 2.526 & 0.169 & 0.552 & Neutral evolution in the Musa Copia 25 clade \\
\hline
\end{tabular}

Critical values of $\mathrm{X}^{2}, 1 \mathrm{df}{ }^{*}: 3.84 ; * * 6.63 ; 2 \Delta \ell=2\left(1_{1}-1_{0}\right)$ 


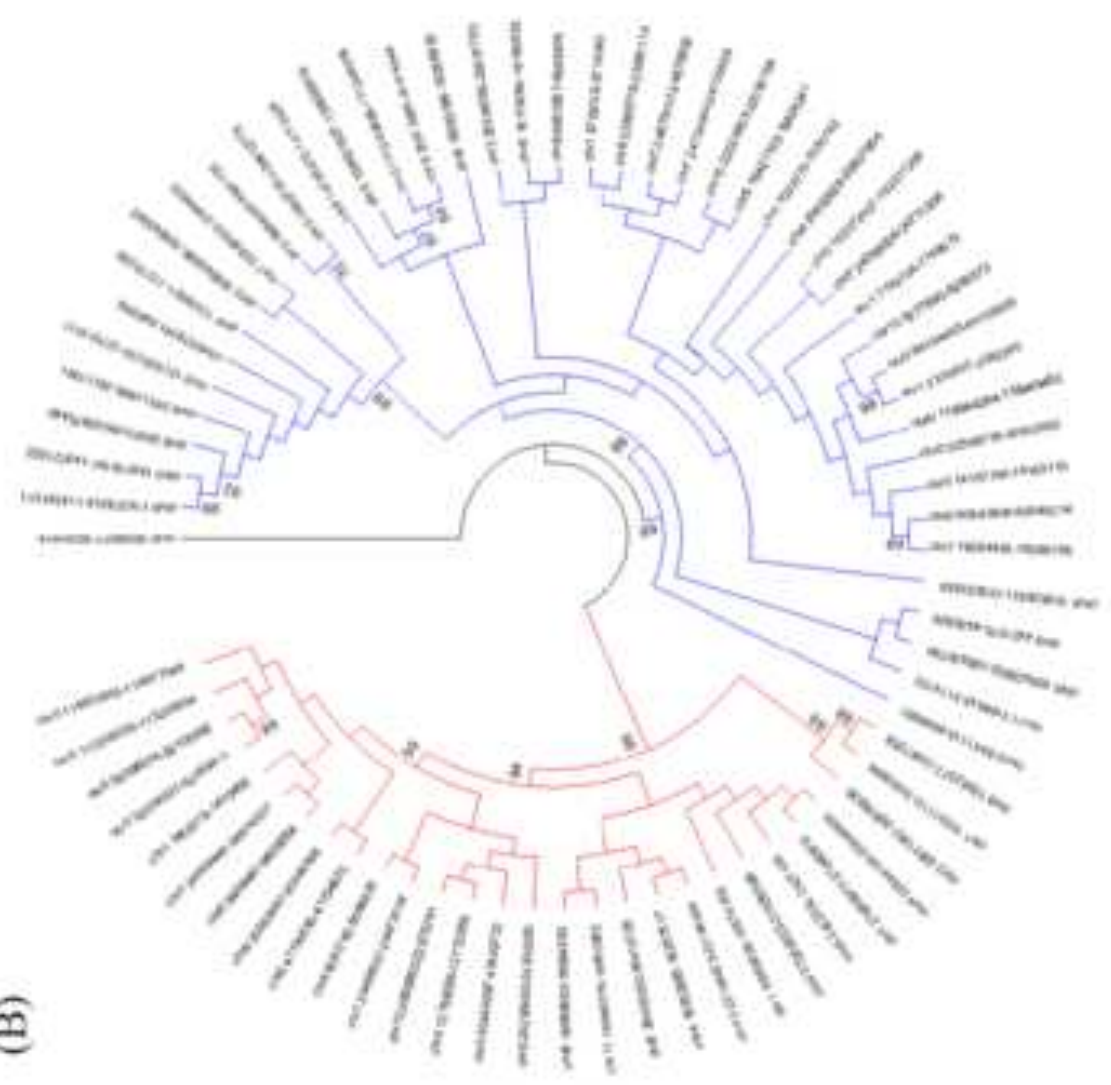

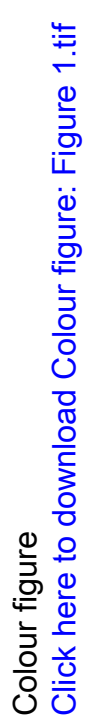

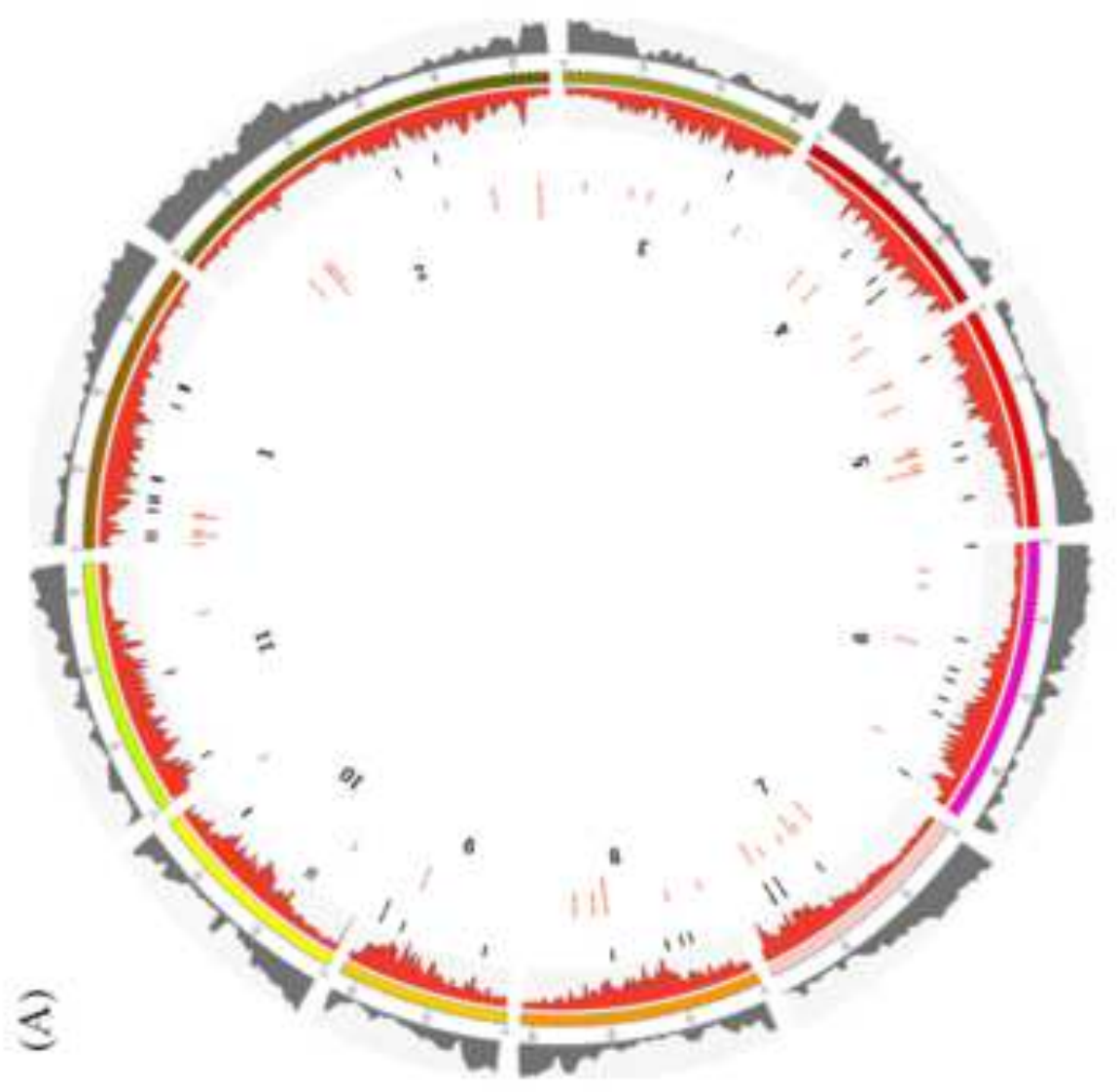




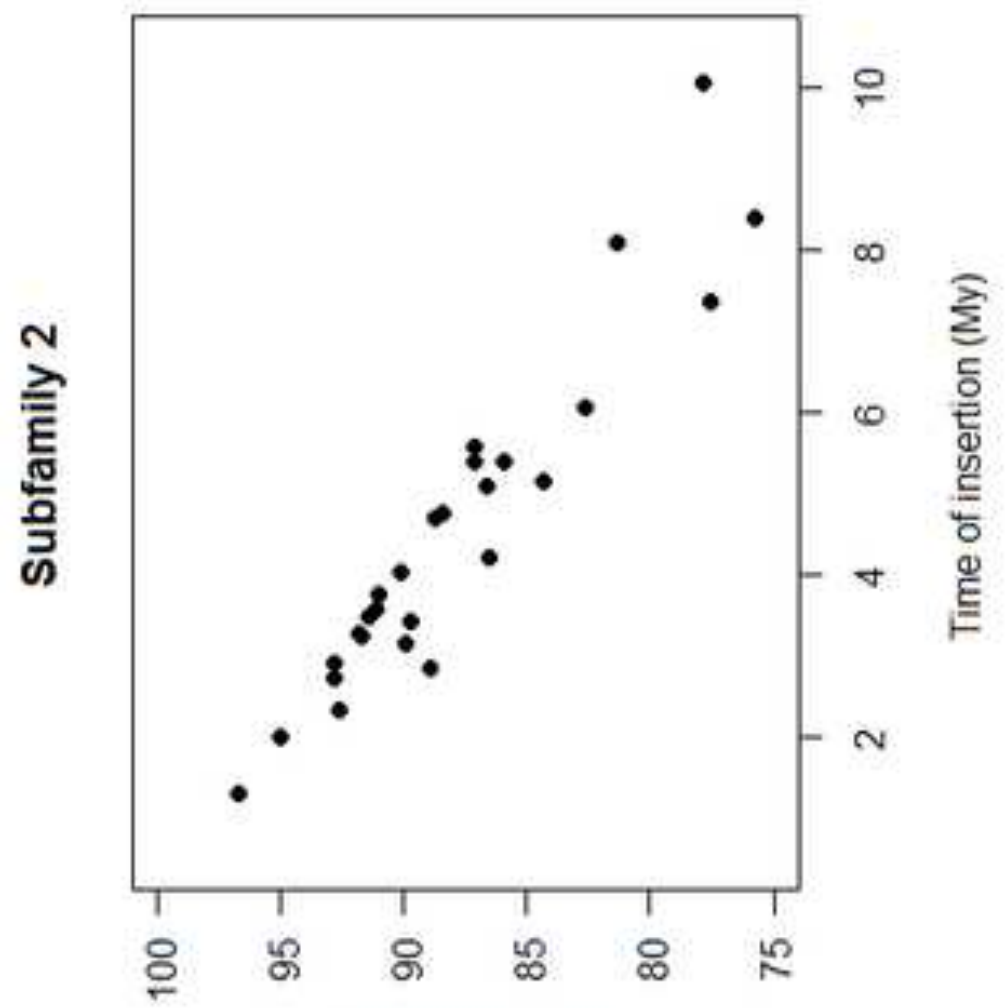

(\%) Nqguap| әp!̣oejonN

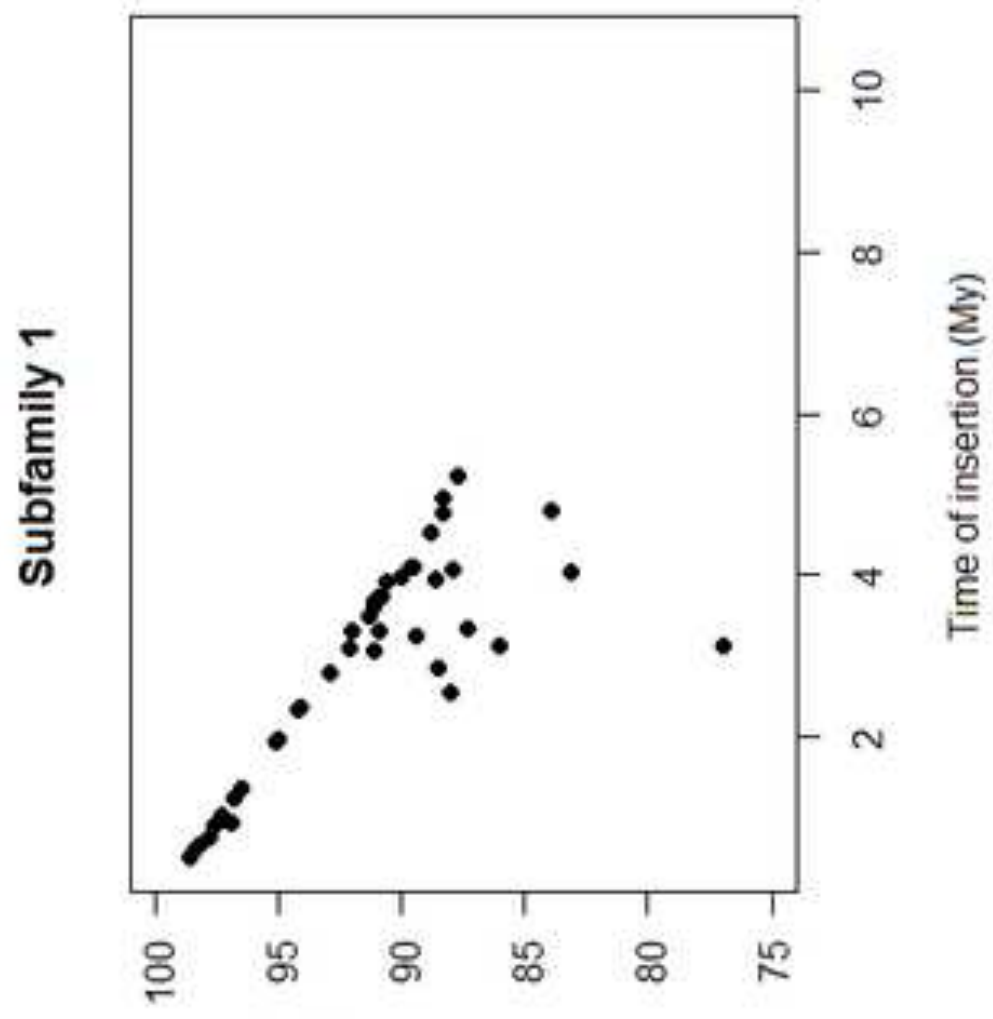

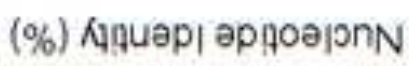






픔

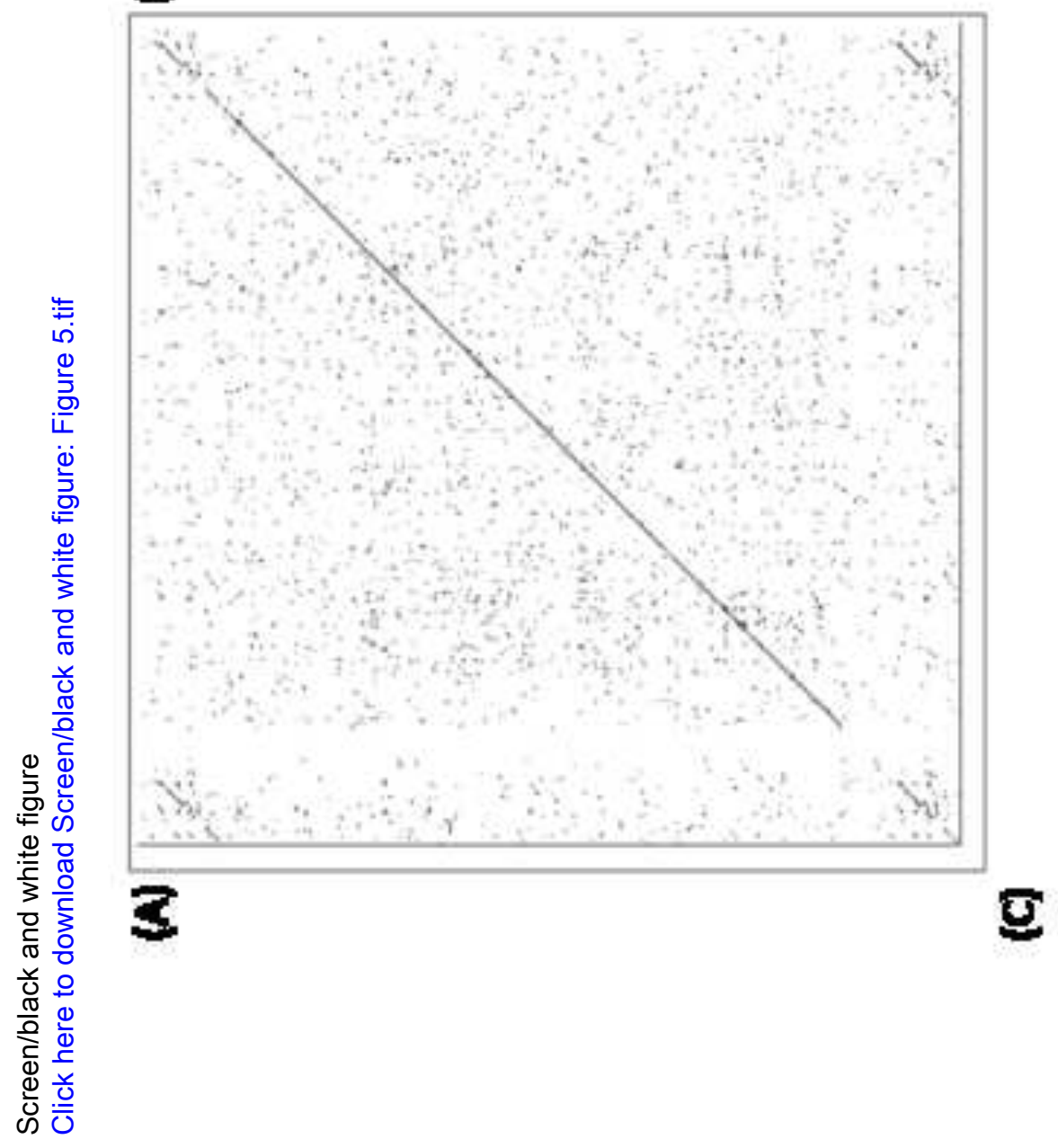

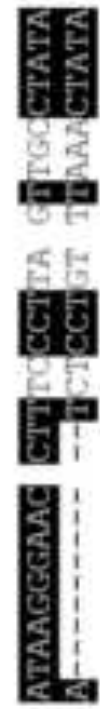

탕하

国贯

Exa

(x)
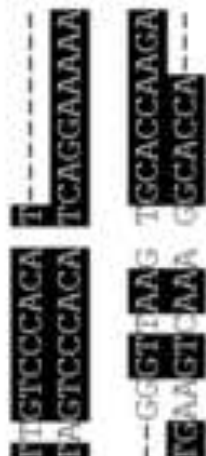

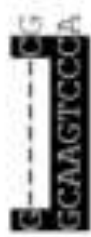

발빕

불

달둥

웣둔

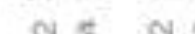

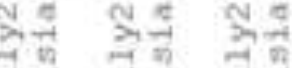

령

달 간

量

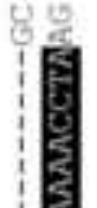

묘리

Ex:

tert

国直

동

프ㄴㅕㅠ

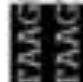

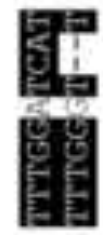

분연 분

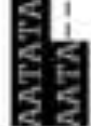

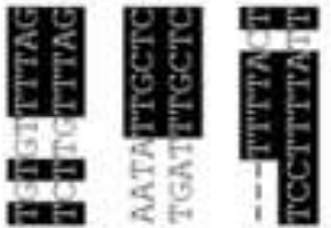
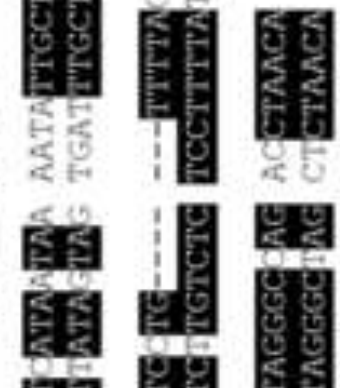

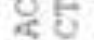

타리

타르

불

은읨

난

域

뎌열

울일

로

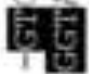

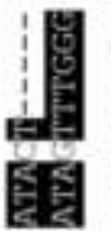

볼

졉

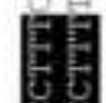

다을

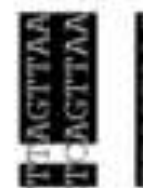

5 \&

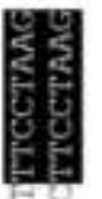

합하영

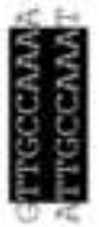

道 5 ह5

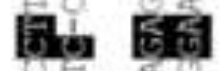

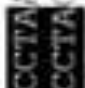

है

탄붕

불

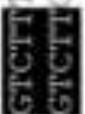

바티

반단

턱 들
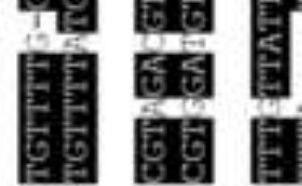

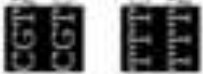

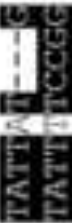

으릴

방붕



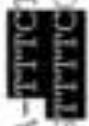
$=$

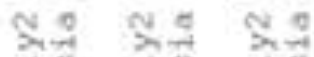

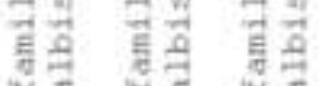

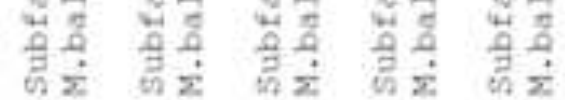






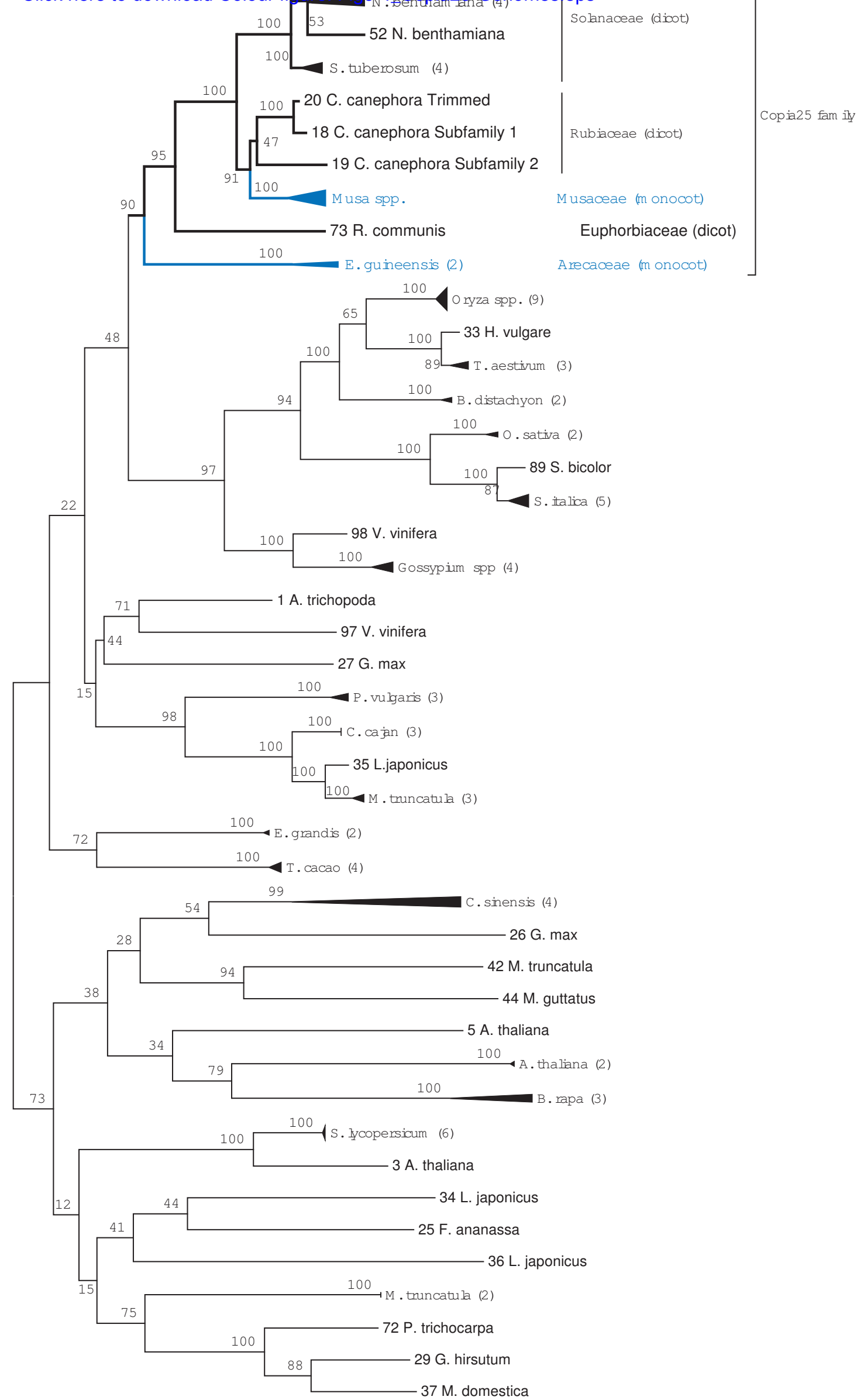


Colour figure

Click here to download Colour figure: Fig4-5_Rub-Gen.eps

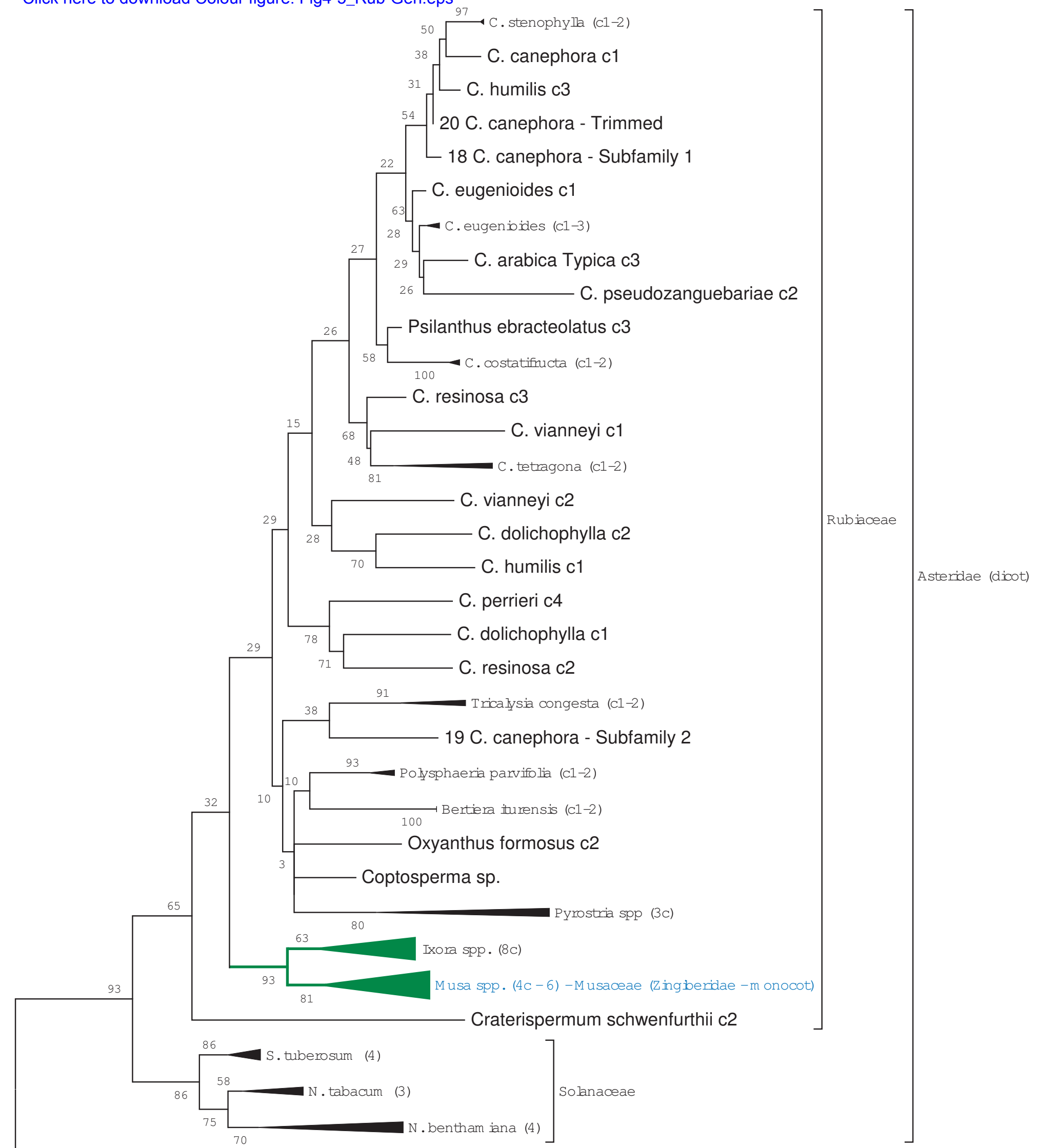

73 R. communis - Euphorbiaceae (Rosidae - dicot) 


\section{Supplementary material}

Click here to download Supplementary material: Final_PMB_SM.docx 